\title{
A Gas-Kinetic Unified Algorithm for Non-Equilibrium Polyatomic Gas Flows Covering Various Flow Regimes
}

\author{
Wen-Qiang $\mathrm{Hu}^{1}$, Zhi-Hui Li ${ }^{1,2, *}$, Ao-Ping Peng ${ }^{2}$ and Xin-Yu Jiang ${ }^{2}$ \\ ${ }^{1}$ National Laboratory for Computational Fluid Dynamics, Beijing University of \\ Aeronautics and Astronautics, Beijing 100191, China. \\ ${ }^{2}$ Hypervelocity Aerodynamics Institute, China Aerodynamics Research and \\ Development Center, Mianyang 621000, China.
}

Received 30 June 2020; Accepted (in revised version) 10 November 2020

\begin{abstract}
In this paper, a gas-kinetic unified algorithm (GKUA) is developed to investigate the non-equilibrium polyatomic gas flows covering various regimes. Based on the ellipsoidal statistical model with rotational energy excitation, the computable modelling equation is presented by unifying expressions on the molecular collision relaxing parameter and the local equilibrium distribution function. By constructing the corresponding conservative discrete velocity ordinate method for this model, the conservative properties during the collision procedure are preserved at the discrete level by the numerical method, decreasing the computational storage and time. Explicit and implicit lower-upper symmetric Gauss-Seidel schemes are constructed to solve the discrete hyperbolic conservation equations directly. Applying the new GKUA, some numerical examples are simulated, including the Sod Riemann problem, homogeneous flow rotational relaxation, normal shock structure, Fourier and Couette flows, supersonic flows past a circular cylinder, and hypersonic flow around a plate placed normally. The results obtained by the analytic, experimental, direct simulation Monte Carlo method, and other measurements in references are compared with the GKUA results, which are in good agreement, demonstrating the high accuracy of the present algorithm. Especially, some polyatomic gas non-equilibrium phenomena are observed and analysed by solving the Boltzmann-type velocity distribution function equation covering various flow regimes.
\end{abstract}

AMS subject classifications: 35Q20, 76P05, 82C40

Key words: Gas-kinetic unified algorithm, polyatomic gas, ellipsoidal statistical model, conservative discrete velocity ordinate method, implicit scheme.

*Corresponding author. Email addresses: vincenthu@buaa.edu.cn (W.-Q. Hu), zhli0097@x263.net (Z.-H. Li), pengaoping@163.com (A.-P. Peng), janxy1987@163.com (X.-Y. Jiang) 


\section{Nomenclature}

\begin{tabular}{|c|c|}
\hline$\alpha$ & energy-dependent deflection-angle exponent \\
\hline$\beta$ and $\theta$ & introduced relaxation parameter \\
\hline$\delta$ & non-translational DoF of the gas \\
\hline$\eta$ & accommodation coefficient of solid wall \\
\hline$\lambda$ & mean free path \\
\hline$v$ & collision frequency \\
\hline$\sigma$ and $\zeta$ & discrete velocity indexes in the $V_{x}$ - and $V_{y}$-direction \\
\hline$\tau$ & viscous stress tensor \\
\hline$\chi$ & temperature exponent of the coefficient of viscosity \\
\hline$\omega$ & index of the VHS model \\
\hline$A$ & weight of numerical quadrature rule \\
\hline$e_{t r}$ and $e_{r o t}$ & energies of translational motion and rotational structure \\
\hline$E$ & total energy \\
\hline$\underset{f}{f S}$ & $\begin{array}{l}\text { gas molecular velocity distribution function } \\
\text { equilibrium distribution function }\end{array}$ \\
\hline$f_{0}$ & gas particle distribution in the $(\overrightarrow{\mathbf{r}}, \overrightarrow{\mathbf{V}})$ phase space \\
\hline$f_{1}$ & the rotational energy density distribution \\
\hline$I$ & internal energy parameter \\
\hline$k$ & Boltzmann constant \\
\hline$m$ & gas molecular mass \\
\hline$n$ & particle number density \\
\hline$P$ & pressure \\
\hline $\operatorname{Pr}$ & Prandtl number \\
\hline$R$ & gas constant \\
\hline$T_{o v}, T_{t r}, T_{r o t}$ and $T_{r e l}$ & $\begin{array}{l}\text { overall, translational, rotational and relaxation temperature } \\
\text { rotational collision number }\end{array}$ \\
\hline$\Theta$ & opposite of the stress tensor \\
\hline $\mathcal{T}$ & corrected tensor \\
\hline $\begin{array}{l}\overrightarrow{\mathbf{q}_{\mathbf{t r}}}, \overrightarrow{\mathbf{q}_{\text {rot }}} \text { and } \overrightarrow{\mathbf{q}} \\
\overrightarrow{\mathbf{r}}=(x, y, z)^{T}\end{array}$ & $\begin{array}{l}\text { translational, rotational and total heat flux vector } \\
\text { space position vector }\end{array}$ \\
\hline $\overrightarrow{\mathbf{U}}=(U, V, W)^{T}$ & flow velocity vector \\
\hline $\overrightarrow{\mathbf{V}}=\left(V_{x}, V_{y}, V_{z}\right)^{T}$ & molecular velocity vector \\
\hline
\end{tabular}

\section{Introduction}

During spacecraft re-entry into atmosphere, various flow regimes can be confronted, e.g., the free molecule flow, rarefied transitional flow, slip and continuum flow regimes [1], where the thermodynamic properties of the gases and the non-equilibrium mechanism 
of flow fields around the spacecraft are quite different [2-4]. Besides, due to the huge distinctions of the geometrical scales at different positions of the spacecrafts, both continuum, transitional and rarefied flow regimes may be encountered in one single case of this flow study $[5,6]$. In addition, for the high-speed high-temperature flow field around the spacecraft during the reentry, different types degrees of freedom (DoF) of the gas molecules have been excited at a certain extent, such as the translational, rotational, vibrational and electronic DOFs, which may arise the energy exchanges, leading some complex chemical and ionization reactions among the molecules and atoms [7]. Correspondingly, the macroscopic dynamics of the gas and the flow states present complex nonequilibrium phenomena. And according to the multiple scale between the flow characteristic time and the gas-kinetic characteristic relaxation time, these non-equilibrium flows can be categorized into the translational-rotational, vibrational-chemical and ionizationradiation non-equilibrium $[8,9]$ etc.. In order to study these complex and mixed nonequilibrium flows correctly, constructing and developing an unified algorithm for high rarefied to continuum polyatomic gas flows has become more and more necessary and important in recent years, which may be contributed to the aerospace engineering.

The Boltzmann equation has been seen to describe the molecular transport phenomena for the full spectrum flow regimes and act as the main foundation for the complex gas dynamic studies [10], which can model the time evolution of the gas molecular velocity distribution function (VDF) from non-equilibrium to equilibrium state. However, the analytical solutions of this equation are possible only for some simple problems, and it may encounter formidable mathematical difficulties for the analysis of the gas dynamic phenomena covering various flow regimes [11]. For example, the computational cost of the Boltzmann collision operator is usually of the order of $N_{V}^{7}$ for the monatomic gases, where $N_{V}$ is the number of discrete velocity ordinate grid points in each velocity direction [12]. For the polyatomic gases, the problem becomes more unbearable when the internal degrees of freedom, such as the rotation and vibration, are considered in the framework of the Wang-Chang-Uhlenbeck (WCU) equation $[13,14]$.

One common and feasible strategy to overcome the difficulty of huge amount of the computational cost is by means of the nonlinear kinetic model equations. In the past decades, various kinetic model equations have been proposed, e.g., the Bhatnagar-GrossKrook (BGK) model [15], Shakhov model [16], and ellipsoidal statistical (ES) model [17], which resemble the original Boltzmann equation and concern about the various order of moments, and the mass, momentum, and energy conservation laws. In addition, considering the excited internal energy levels of the gas molecules, the Rykov model [18] for the diatomic gases can be seen as the extension of the Shakhov model for monatomic gas. Andries et al. [19] and Brull et al. [20] extend the ES model into a modified version for the polyatomic gases, which add the internal energy parameter as an independent variable into the molecular VDF. Bernard et al. [21] present a new model of BGK type for polyatomic gases, which incorporates the different relaxation rates of translational, rotational and/or vibrational modes characterizing polyatomic molecules. Dauvois et al. [22] propose an extension of the ES-BGK model to account for discrete levels of vibrational 
energy in a rarefied polyatomic gas, which contains parameters that allow to fit almost arbitrary values for the Prandtl number and the relaxation times of rotational and vibrational energies. Based on a system of kinetic ES-BGK equations, Klingenberg et al. [23] give a consistent kinetic model for a two-component mixture of polyatomic molecules.

Instead of solving the full Boltzmann equation, it may be a more economical and efficient way for the simulations of the complex gas flows over a wide range of Knudsen numbers to solves these kinetic model equations using suitable numerical methods. During past decades, various methods have been developed to simulate these flows based on the kinetic theories, such as the lattice Boltzmann method (LBM) [24-26], the kinetic flux vector splitting (KFVS) schemes [27], the gas kinetic scheme (GKS) [28-30], the gaskinetic unified algorithm (GKUA) [31,32], the moment methods [33], and the unified gas kinetic scheme (UGKS) [34,35] etc.

Among these methods, the GKUA have been successfully used to study hypersonic reentry aerothermodynamics around kinds of space vehicles and micro-scale flows involved in MEMS devices [36,37]. The GKUA has unified expressions on the molecular collision relaxing parameter and the local equilibrium distribution function, which are associated with the macroscopic flow variables, the gas viscosity transport coefficient, the thermodynamic effect, the molecular power law, molecular models, and the flow state controlling parameter $\mathrm{Kn}$ from various flow regimes. The GKUA is firstly developed and employed systematically the discrete velocity ordinate method (DVOM) to solve the unified non-equilibrium VDF equation for three-dimensional complex flows at arbitrary Mach numbers covering various flow regimes [5]. The VDF equation from the kinetic models is numerically discretized in the velocity space by the DVOM with the conservation constraints of summation invariants and $\mathrm{H}$-theorem on the Boltzmann equation. Through the numerical quadrature rules, the macroscopic flow variables can be obtained by the moments of the VDF over the discrete velocity space. Applying the high-efficiency integral methods, the discrete velocity ordinate points in the velocity space are not very fine, and the DVOM with the wide needed truncated velocity interval for the high Mach number three-dimensional flows have been presented and applied in practice [4]. It has been indicated from Refs. $[38,39]$ that the DVOM is different from the conventional discrete velocity ordinate method [40] based on the Maxwell equilibrium distribution for low-speed flow.

In order to study the non-equilibrium flow of spacecraft re-entry into the atmosphere covering various flow regimes, the gas-kinetic unified algorithm will be extended and considered into the simulations of the translational-rotational non-equilibrium flow in this work. Based on the ES model with rotational energy excitation and regarding the rotational energy as an independent variable of the molecular VDF, we will develop a new version of the GKUA for polyatomic gas flows in rotational non-equilibrium state. Hereby, the remaining part of this paper will be organized as follows. The kinetic modeling of polyatomic gases for the Boltzmann-type velocity distribution function equation will be given briefly in Section 2, including the explicit expressions of the model in multidimension physical space, and the deterministic technology of the introduced relaxation 
parameters. Numerical procedures of the GKUA will be illustrated in Section 3, including the discrete velocity ordinate method, the lower-upper symmetric Gauss-Seidel (LUSGS) implicit scheme, and the initialization and boundary condition procedures. Section 4 presents some numerical test cases and the analysis discussions. The last section is the concluding remarks.

\section{Kinetic modelling of polyatomic gases in rotational non-equilibrium state}

Generally, the monatomic gases can be seen as a collection of particles characterized by their space position $\overrightarrow{\mathbf{r}}=(x, y, z)^{T}$ and velocity $\overrightarrow{\mathbf{V}}=\left(V_{x}, V_{y}, V_{z}\right)^{T}$, whose relevant mathematical model is then the Boltzmann equation [41],

$$
\frac{\partial f}{\partial t}+\overrightarrow{\mathbf{V}} \cdot \nabla_{\overrightarrow{\mathbf{r}}} f+\overrightarrow{\mathbf{F}} \cdot \nabla_{\overrightarrow{\mathbf{v}}} f=Q(f, f)
$$

where $f$ is the molecular VDF depending on the space position $\overrightarrow{\mathbf{r}}$, molecular velocity $\overrightarrow{\mathbf{V}}$ and time $t, \overrightarrow{\mathbf{F}}$ is the vector of the external force, and $Q(f, f)$ is a complex integrodifferential term, which describes the binary collision between particles.

The extension to polyatomic gases can be achieved by considering the internal energy, such as the rotational or vibrational energy, which can be expressed as follows [19],

$$
\varepsilon_{\text {int }}=I^{2 / \delta},
$$

where $I$ is the internal energy parameter which is non-negative, i.e., $I \in \mathbb{R}^{+}$, and $\delta$ is the measure of excitation of internal energy levels and non-translational DoF of the gas. Typically, for polyatomic gases, some internal DoF are only partially excited, with the degree of excitation determined by temperature. Therefore, $\delta$ is not an integer, but a continuous function of temperature, i.e., $\delta=\delta(T)$, that has integer values only for fully excited internal DoF.

In this paper, we consider polyatomic gases in which the vibrational degrees of freedom are not excited and the rotational degrees of freedom can be treated classically. For this case, the gas molecule has three translational degrees of freedom and $\delta$ rotational degrees of freedom, where $\delta=2$ for diatomic and linear polyatomic gases and $\delta=3$ for nonlinear polyatomic gases [12].

Hereby, the governing equation of the polyatomic molecular transport phenomena from full spectrum of flow regimes in the view of micro-mechanics can also be described by the Boltzmann equation (2.1), where the molecular distribution function is rewritten as $f=f(t, \overrightarrow{\mathbf{r}}, \overrightarrow{\mathbf{V}}, I)$ with $(t, \overrightarrow{\mathbf{r}}, \overrightarrow{\mathbf{V}}, I) \in \mathbb{R}^{+} \times \mathbb{R} \times \mathbb{R} \times \mathbb{R}^{+}$. 


\subsection{Ellipsoidal statistical model with rotational energy excitation}

Ignoring the effect of the external forces, the ellipsoidal statistical model equation with the excitation of internal energy levels (ES-int) for polyatomic gases $[19,20]$ has the following form, which can be seen as the extension of the ES model for monatomic gas [17],

$$
\frac{\partial f}{\partial t}+\overrightarrow{\mathbf{V}} \cdot \nabla \overrightarrow{\mathbf{r}} f=v\left(f^{E S}-f\right)
$$

where $v$ is a constant parameter which is called the collision frequency and determined by the viscosity, and $f^{E S}$ is the equilibrium distribution function, whose specific form is given by the expression (2.10).

Under the notation

$$
\langle\langle\Psi f\rangle\rangle=\int_{\overrightarrow{\mathbf{V}} \in \mathbb{R}^{3}, I \in \mathbb{R}^{+}} \Psi f(t, \overrightarrow{\mathbf{r}}, \overrightarrow{\mathbf{V}}, I) d \overrightarrow{\mathbf{V}} d I,
$$

the relations between distribution function and macroscopic quantities are defined as

$$
\begin{aligned}
& n(\overrightarrow{\mathbf{r}}, t)=\langle\langle f\rangle\rangle, \\
& \overrightarrow{\mathbf{U}}(\overrightarrow{\mathbf{r}}, t)=\langle\langle\overrightarrow{\mathbf{V}} f\rangle\rangle, \\
& E(\overrightarrow{\mathbf{r}}, t)=\left\langle\left\langle\left(\frac{1}{2}|\overrightarrow{\mathbf{V}}|^{2}+I^{\frac{2}{\delta}}\right) f\right\rangle\right\rangle=\frac{1}{2} n|\overrightarrow{\mathbf{U}}|^{2}+n e,
\end{aligned}
$$

where $\Psi$ is an arbitrary function, $n$ is the particle number density, $\overrightarrow{\mathbf{U}}=(U, V, W)^{T}$ is the flow velocity, $E$ is the total energy, and $e$ is the specific internal energy, which can be divided here in two parts, the energy of translational motion $e_{t r}$ and the energy associated with the rotational structure $e_{\text {rot }}$,

$$
e=\frac{E}{\rho}-\frac{1}{2}|\overrightarrow{\mathbf{U}}|^{2}=e_{t r}+e_{r o t}, \quad e_{t r}=\frac{1}{2 n}\left\langle\left\langle|\overrightarrow{\mathbf{V}}-\overrightarrow{\mathbf{U}}|^{2} f\right\rangle\right\rangle, \quad e_{r o t}=\frac{1}{n}\left\langle\left\langle I^{\frac{2}{\delta}} f\right\rangle\right\rangle .
$$

The classical equipartition theorem states that in full thermal equilibrium, and with $\delta$ fully excited internal DoF, each DoF contributes an energy of $R T / 2$ to the energy of a particle [20]. Hence, these energies can be associated with their corresponding temperature $T_{o v}, T_{t r}$ and $T_{\text {int }}$, i.e.,

$$
e=\frac{3+\delta}{2} R T_{o v}, \quad e_{t r}=\frac{3}{2} R T_{t r}, \quad e_{r o t}=\frac{\delta}{2} R T_{r o t},
$$

where $R$ is the gas constant, calculated by the fraction of the Boltzmann constant $k$ and the molecular mass $m$.

As same as the treatment of the original ES model, we introduce the opposite of the stress tensor of the following form,

$$
\boldsymbol{\Theta}=\frac{1}{n}\langle\langle\overrightarrow{\mathbf{C}} \otimes \overrightarrow{\mathbf{C}} f\rangle\rangle,
$$


where $\overrightarrow{\mathbf{C}}=\overrightarrow{\mathbf{V}}-\overrightarrow{\mathbf{U}}$ is the peculiar velocity, and the symbol $\otimes$ represents the operation of the tensor product. Another two relaxation parameters $\beta$ and $\theta$ are introduced to define the relaxation temperature,

$$
T_{r e l}=\theta T_{o v}+(1-\theta) T_{r o t},
$$

and the corrected tensor

$$
\mathcal{T}=(1-\theta)\left[(1-\beta) R T_{t r} \mathbf{I}_{\mathbf{d}}+\beta \boldsymbol{\Theta}\right]+\theta R T_{o v} \mathbf{I}_{\mathbf{d}}
$$

where $\mathbf{I}_{\mathbf{d}}$ is the unit tensor. Heretofore, the explicit and specific expression of the equilibrium distribution function $f^{E S}$ can be defined as,

$$
f^{E S}=\frac{n \Lambda_{\delta}}{\sqrt{\operatorname{det}(2 \pi \mathcal{T})}\left(R T_{r e l}\right)^{2 / \delta}} \exp \left(-\frac{1}{2} \overrightarrow{\mathbf{C}} \cdot \mathcal{T}^{-1} \cdot \overrightarrow{\mathbf{C}}-\frac{I^{2 / \delta}}{R T_{r e l}}\right),
$$

with

$$
\Lambda_{\delta}^{-1}=\int_{\mathbb{R}^{+}} \exp \left(-I^{2 / \delta}\right) d I=\Gamma\left(1+\frac{\delta}{2}\right)=\frac{\delta}{2} \Gamma\left(\frac{\delta}{2}\right),
$$

where $\Gamma$ is the Gamma function, calculated as $\Gamma(1 / 2)=\sqrt{\pi}, \Gamma(1)=1$ and $\Gamma(1+x)=x \Gamma(x)$.

In order to reduce the amount of computations, a considerable method can be realized according to the fact that the constructed model kinetic equation can be averaged over the rotational energy parameter $I$. Using integration with respect to $I$, we proceed from the molecular distribution function $f(t, \overrightarrow{\mathbf{r}}, \overrightarrow{\mathbf{v}}, I)$ to the new functions,

$$
\begin{aligned}
& f_{0}(t, \overrightarrow{\mathbf{r}}, \overrightarrow{\mathbf{v}})=\int_{\mathbb{R}^{+}} f(t, \overrightarrow{\mathbf{r}}, \overrightarrow{\mathbf{V}}, I) d I, \\
& f_{1}(t, \overrightarrow{\mathbf{r}}, \overrightarrow{\mathbf{v}})=\int_{\mathbb{R}^{+}} e_{r o t} f(t, \overrightarrow{\mathbf{r}}, \overrightarrow{\mathbf{V}}, I) d I .
\end{aligned}
$$

Here, $f_{0}$ is the gas particle distribution in the $(\overrightarrow{\mathbf{r}}, \overrightarrow{\mathbf{v}})$ phase space and $f_{1}$ is the rotational energy density distribution in this space. Correspondingly, the model equation (2.3) can be transformed into the following forms,

$$
\frac{\partial f_{i}}{\partial t}+\overrightarrow{\mathbf{V}} \cdot \nabla_{\overrightarrow{\mathbf{r}}} f_{i}=v\left(f_{i}^{E S}-f_{i}\right), \quad i=0,1
$$

with

$$
f_{0}^{E S}=\frac{n}{(2 \pi)^{3 / 2} \sqrt{\operatorname{det}(\mathcal{T})}} \exp \left(-\frac{1}{2} \overrightarrow{\mathbf{C}} \cdot \mathcal{T}^{-1} \cdot \overrightarrow{\mathbf{C}}\right), \quad f_{1}^{E S}=\frac{\delta}{2} R T_{\text {rel }} f_{0}^{E S} .
$$

And the collision frequency $v$ has the following forms [?], which considers the molecular interaction and the rarefaction degree of gas flows from rarefied to continuum,

$$
v=\operatorname{Pr} \cdot \frac{4 \alpha(5-2 \omega)(7-2 \omega)}{5(\alpha+1)(\alpha+2)} \sqrt{\frac{R}{2 \pi}} \cdot \frac{T_{r e f}^{\chi-\frac{1}{2}}}{n_{r e f}} \cdot \frac{1}{\lambda_{r e f}} \cdot \frac{n}{T \chi-1},
$$


Table 1: Characteristic variables of each physical quantity for non-dimensionalizing unified simplified VDF equation.

\begin{tabular}{||cc||}
\hline Physical Quantity & Characteristic variable \\
\hline Length $L_{r e f}$ & $L_{r e f}$ \\
Density $n_{r e f}$ & $n_{r e f}$ \\
Temperature $T_{r e f}$ & $T_{r e f}$ \\
Speed $c_{r e f}$ & $\sqrt{2 R T_{r e f}}$ \\
Time $t_{r e f}$ & $L_{r e f} / c_{r e f}$ \\
Collision Frequency $v_{r e f}$ & $1 / t_{r e f}$ \\
Pressure $P_{r e f}$ & $\frac{1}{5} \cdot m n_{r e f} c_{r e f}^{2} / 2$ \\
Heat Flux $q_{r e f}$ & $m n_{r e f} c_{r e f}^{3} / 2$ \\
Distribution Function $f_{0, \infty}$ & $n_{r e f} / c_{r e f}^{3}$ \\
Distribution Function $f_{1, \infty}$ & $n_{r e f} / 2 c_{r e f}$ \\
Opposite of Stress Tensor $\boldsymbol{\Theta}_{r e f}$ & $c_{r e f}^{2} / 2$ \\
Corrected Tensor $\mathcal{T}_{r e f}$ & $c_{r e f}^{2} / 2$ \\
\hline
\end{tabular}

where $\alpha$ is the energy-dependent deflection-angle exponent which denotes the index of the variable soft sphere (VSS) molecular model [42], $\omega$ is the index of the VHS model [43], $T_{r e f}$ and $n_{r e f}$ are the reference temperature and number density, respectively, which are set as the free-stream values generally, $\chi$ is the temperature exponent of the coefficient of viscosity, $\lambda_{r e f}$ is the reference mean free path, $P r$ is the Prandtl number which has the following relation with the two additional introduced parameters $\beta$ and $\theta$,

$$
\operatorname{Pr}=\frac{1}{1-\beta+\theta \beta}
$$

and other parameters are as same meaning as mentioned before.

Following the characteristic variables in Table 1, the non-dimensional unified VDF equations presented in the Cartesian coordinates are of the same expressions as Eq. (2.12), while

$$
\begin{aligned}
& f_{0}^{E S}=\frac{n}{\pi^{\frac{3}{2}} \sqrt{\operatorname{det}(\mathcal{T})}} \exp \left(-\overrightarrow{\mathbf{C}} \cdot \mathcal{T}^{-1} \cdot \overrightarrow{\mathbf{C}}\right), \quad f_{1}^{E S}=\frac{\delta}{2} T_{r e l} f_{0}^{E S}, \\
& \nu=\operatorname{Pr} \cdot \frac{2 \alpha(5-2 \omega)(7-2 \omega)}{5(\alpha+1)(\alpha+2) \sqrt{\pi}} \cdot \frac{n T^{1-\chi}}{K n}, \quad \operatorname{Pr}=\frac{1}{1-\beta+\theta \beta}, \quad K n=\frac{\lambda_{r e f}}{L}, \\
& \mathcal{T}_{i j}=(1-\theta)\left[(1-\beta) T_{t r} \Delta_{i j}+\beta \boldsymbol{\Theta}_{i j}\right]+\theta T_{o v} \Delta_{i j} \\
& \operatorname{det}(\mathcal{T})=\mathcal{T}_{x x} \mathcal{T}_{y y} \mathcal{T}_{z z}-\mathcal{T}_{x y}^{2} \mathcal{T}_{z z}-\mathcal{T}_{x z}^{2} \mathcal{T}_{y y}-\mathcal{T}_{y z}^{2} \mathcal{T}_{x x}+2 \mathcal{T}_{x y} \mathcal{T}_{x z} \mathcal{T}_{y z},
\end{aligned}
$$




$$
\overrightarrow{\mathbf{C}} \cdot \mathcal{T}^{-1} \cdot \overrightarrow{\mathbf{C}}=\frac{1}{\operatorname{det}(\mathcal{T})}\left(\begin{array}{l}
C_{x}^{2}\left(\mathcal{T}_{y y} \mathcal{T}_{z z}-\mathcal{T}_{y z}^{2}\right)+C_{y}^{2}\left(\mathcal{T}_{x x} \mathcal{T}_{z z}-\mathcal{T}_{x z}^{2}\right)+C_{z}^{2}\left(\mathcal{T}_{x x} \mathcal{T}_{y y}-\mathcal{T}_{x y}^{2}\right) \\
+2 C_{x} C_{y}\left(\mathcal{T}_{x z} \mathcal{T}_{y z}-\mathcal{T}_{x y} \mathcal{T}_{z z}\right)+2 C_{x} C_{z}\left(\mathcal{T}_{x y} \mathcal{T}_{y z}-\mathcal{T}_{x z} \mathcal{T}_{y y}\right) \\
+2 C_{y} C_{z}\left(\mathcal{T}_{x y} \mathcal{T}_{x z}-\mathcal{T}_{y z} \mathcal{T}_{x x}\right)
\end{array}\right)
$$

where $K n$ is the Knudsen number, $\Delta_{i j}$ is the Kronecker delta symbol, and the constant $\checkmark$ is introduced as the dimensionless adjustment parameter in order to unify different relations of the dimensionless density, temperature and pressure in some previous literatures, which is generally set as unit. All of the variables in the above expressions and in all following equations are non-dimensional, if it is not mentioned especially.

All of the considering macroscopic flow variables, e.g., the number density of the gas $n$, the flow velocity $\overrightarrow{\mathbf{U}}$, the four type temperature $T_{t r}, T_{r o t}, T_{o v}$ and $T_{r e l}$, the pressure $P$, the viscous stress tensor $\tau$ and the three type heat flux vector $\overrightarrow{\mathbf{q}_{\mathrm{tr}}}, \overrightarrow{\mathbf{q}_{\text {rot }}}$ and $\overrightarrow{\mathbf{q}}$, can be calculated from the integrated moments of the VDF over the molecular velocity space,

$$
\begin{aligned}
& n(\overrightarrow{\mathbf{r}}, t)=\int_{\mathbb{R}^{3}} f_{0} d \overrightarrow{\mathbf{V}}, \\
& U_{i}(\overrightarrow{\mathbf{r}}, t)=\frac{1}{n} \int_{\mathbb{R}^{3}} V_{i} f_{0} d \overrightarrow{\mathbf{V}} \\
& T_{t r}(\overrightarrow{\mathbf{r}}, t)=\frac{2}{3 n} \int_{\mathbb{R}^{3}}|\overrightarrow{\mathbf{C}}|^{2} f_{0} d \overrightarrow{\mathbf{V}}, \quad T_{r o t}(\overrightarrow{\mathbf{r}}, t)=\frac{2}{\delta n} \int_{\mathbb{R}^{3}} f_{1} d \overrightarrow{\mathbf{V}}, \\
& T_{o v}(\overrightarrow{\mathbf{r}}, t)=\frac{1}{3+\delta}\left(3 T_{t r}+\delta T_{r o t}\right), \quad T_{r e l}(\overrightarrow{\mathbf{r}}, t)=\theta T_{o v}+(1-\theta) T_{r o t}, \\
& P(\overrightarrow{\mathbf{r}}, t)=\varsigma n T_{o v}, \quad \mathbf{\Theta}_{i j}=\frac{2}{n} \int_{\mathbb{R}^{3}} C_{i} C_{j} f_{0} d \overrightarrow{\mathbf{V}}, \quad \tau_{i j}(\overrightarrow{\mathbf{r}}, t)=-\varsigma n \mathbf{\Theta}_{i j}+P \boldsymbol{\Delta}_{i j}, \\
& q_{t r, i}(\overrightarrow{\mathbf{r}}, t)=\int_{\mathbb{R}^{3}} C_{i}|\overrightarrow{\mathbf{C}}|^{2} f_{0} d \overrightarrow{\mathbf{V}}, \quad q_{r o t, i}(\overrightarrow{\mathbf{r}}, t)=\int_{\mathbb{R}^{3}} C_{i} f_{1} d \overrightarrow{\mathbf{V}}, \quad q_{i}=q_{t r, i}+q_{r o t, i},
\end{aligned}
$$

where the subscripts $i$ and $j$ each range from 1 to 3 , which are identified with components along the $x, y$ and $z$ axes, respectively.

If we define the following three vectors,

$$
\Psi=\left(\begin{array}{cc}
\frac{1}{\vec{V}} & 0 \\
|\overrightarrow{\mathbf{V}}|^{2} & 1
\end{array}\right)
$$

one can obtain the following expressions by virtue of the relations between the molecular VDF and the macroscopic flow variables,

$$
\int_{\overrightarrow{\mathbf{v}} \in \mathbb{R}^{3}} \Psi\left(\begin{array}{c}
f_{0} \\
f_{1}
\end{array}\right) d \overrightarrow{\mathbf{V}}=\left(\begin{array}{c}
n \\
n \overrightarrow{\mathbf{U}} \\
\frac{3}{2} n T_{t r}+n|\overrightarrow{\mathbf{U}}|^{2}+\frac{\delta}{2} n T_{\text {rot }}
\end{array}\right)
$$


From Eq. (2.15), one can obtain the following integral results about the equilibrium distribution function,

$$
\int_{\overrightarrow{\mathbf{v}} \in \mathbb{R}^{3}} \Psi\left(\begin{array}{c}
f_{0}^{E S} \\
f_{1}^{E S}
\end{array}\right) d \overrightarrow{\mathbf{V}}=\left(\begin{array}{c}
n \vec{n} \\
n|\overrightarrow{\mathbf{U}}|^{2}+\frac{n\left(\mathcal{T}_{x x}+\mathcal{T}_{y y}+\mathcal{T}_{z z}\right)}{2}+\frac{\delta}{2} n T_{\text {rel }}
\end{array}\right) .
$$

Through the definitions of the opposite stress tensor $\boldsymbol{\Theta}$ and corrected tensor $\mathcal{T}$ and coupling with Eq. (2.16c), we have

$$
\boldsymbol{\Theta}_{x x}+\Theta_{y y}+\Theta_{z z}=3 T_{t r} \Rightarrow \mathcal{T}_{x x}+\mathcal{T}_{y y}+\mathcal{T}_{z z}=3\left[(1-\theta) T_{t r}+\theta T_{o v}\right] .
$$

Therefore, the third term of Eq. (2.19) can be calculated into the following form,

$$
\int_{\overrightarrow{\mathbf{v}} \in \mathbb{R}^{3}}\left(|\overrightarrow{\mathbf{V}}|^{2} f_{0}^{E S}+f_{1}^{E S}\right) d \overrightarrow{\mathbf{V}}=n|\overrightarrow{\mathbf{U}}|^{2}+\frac{3}{2} n T_{t r}+\frac{\delta}{2} n T_{\text {rot }}
$$

Subtracting Eq. (2.18) from the identical relations (2.19) and (2.21), Eq. (2.15) satisfies the conservation conditions at each of points in physical space and time, i.e.,

$$
\int_{\overrightarrow{\mathbf{v}} \in \mathbb{R}^{3}} \Psi\left(\begin{array}{c}
f_{0}^{E S}-f_{0} \\
f_{1}^{E S}-f_{1}
\end{array}\right) d \overrightarrow{\mathbf{V}}=0
$$

where $\Psi$ can be seen as the components of the moments on mass, momentum and energy. The conservation conditions mean that the mass, momentum and energy are conserved during molecular collisions in this model.

\subsection{Determination of the relaxation parameters $\beta$ and $\theta$}

According to Ref. [19], the values of relaxation parameters $\beta$ and $\theta$ should be in the following interval,

$$
-\frac{1}{2} \leqslant \beta \leqslant 1, \quad 0 \leqslant \theta \leqslant 1,
$$

when the nonnegative characteristics of the molecular VDFs and the macroscopical temperatures are considered, whose specific mathematical derivations have been presented in Ref. [20]. Besides, in the Chapman-Enskog expansion to the Navier-Stokes system, the second viscosity coefficient $\alpha$ and Prandtl number $\operatorname{Pr}$ can link with these two relaxation parameters [19],

$$
\alpha=\gamma-1-\frac{1-\theta}{\theta}(1-\beta)\left(\frac{5}{3}-\gamma\right), \quad \operatorname{Pr}=\frac{\gamma}{\gamma-1} \frac{R \mu}{\kappa}=\frac{1}{1-\beta+\theta \beta} .
$$

When $\theta$ approaches zero, the model equation (2.15) can be reduced to the original ES model for monatomic gases, where a correct Prandtl number $2 / 3$ is obtained by taking 
$\beta=-1 / 2$. For the diatomic or nonlinear polyatomic gases, the experimental values of the Prandtl number can be found in some references. Hence, considering Eq. (2.14) and unifying the expression, the first strategy to determine the values of $\beta$ and $\theta$ is given as follows,

$$
\beta=-\frac{1}{2}, \quad \theta=1+\frac{\operatorname{Pr}^{-1}-1}{\beta} .
$$

For the nitrogen or oxygen gas, whose specific heat ratio is 1.4, the value of Prandtl number is $5 / 7$ approximatively, and this is obtained with $\beta=-0.5$ and $\theta=0.2$. This value of $\theta$ corresponds to the empirical law that in a polyatomic gas, one collision of particles out of five involves an exchange of internal energy, which is widely used in DSMC simulations.

Another strategy is enlighten by the rotational relaxation process in a homogeneous gas. For a diatomic or nonlinear polyatomic homogeneous gas with different initial translational temperature $T_{t r}$ and rotational temperature $T_{r o t}$, the system will evolve into an equilibrium one with average temperature $T$, which is a constant value. Intermolecular collision will relax $T_{\text {rot }}$ into the average temperature $T$ with a rate related to the collision frequency.

Due to the homogeneous space distribution, which exists no molecular convective movements, the governing equations (2.15) can be reduced as,

$$
\frac{d f_{i}}{d t}=v\left(f_{i}^{E S}-f_{i}\right), \quad i=0,1 .
$$

Integrating the above equation over the whole velocity with weighting factors $|\overrightarrow{\mathbf{C}}|^{2}$ and 1 , respectively, the time evolutions of the translational and rotational energies can be obtained as,

$$
\left\{\begin{array}{l}
\frac{d T_{t r}}{d t}=\theta v\left(T_{o v}-T_{t r}\right), \\
\frac{d T_{r o t}}{d t}=v\left(T_{r e l}-T_{r o t}\right)=\theta v\left(T_{o v}-T_{r o t}\right) .
\end{array}\right.
$$

Comparing with the relaxation process for a homogeneous gas in Ref. [34] and considering the definition of the collision frequency, we can derive the following relation for the accordance,

$$
\theta=\frac{1}{Z \cdot P r}
$$

with

$$
Z=\frac{Z^{\infty}}{1+\left(\pi^{3 / 2} / 2\right) \sqrt{T^{*} / T_{t r}}+\left(\pi+\pi^{2} / 4\right)\left(T^{*} / T_{t r}\right)},
$$

where the parameter $Z$ is the rotational collision number in the Landau-Teller-Jeans type relaxation model, the quantity $Z^{\infty}$ has a fixed value, and $T^{*}$ is the characteristic temperature of intermolecular potential. For the nitrogen gas, the values $Z^{\infty}=23.0$ and $T^{*}=91.5$ are utilized, while $Z^{\infty}=14.4$ and $T^{*}=90.0$ for the oxygen. Besides, the parameter $Z$ can be a constant in some engineering computations, e.g., $Z=5.0$. The numerical validation 
of the relaxation process for a homogeneous gas using our model will be given in Section 4. Coupling Eq. (2.28) with Eq. (2.14), the second strategy of determination can obtain as the following form,

$$
\beta=\frac{Z(1-P r)}{1-Z \cdot P r}, \quad \theta=\frac{1}{Z \cdot P r} .
$$

\subsection{Reduced velocity distribution function equations for the two-dimensional flows}

A considerable reduction in the amount of computations can be achieved through the accurate integration of the VDFs on the velocity components in some directions with appropriate weighting factors. For the two-dimensional problems in the space direction $x$ and $y, \partial_{z}(\bullet)=0$ ( $\bullet$ represents arbitrary status function). Introducing three reduced distribution functions of $x, y, V_{x}, V_{y}$ and $t$ with the following forms,

$$
\begin{aligned}
& g_{0}\left(x, y, V_{x}, V_{y}, t\right)=\int_{\mathbb{R}} f_{0}\left(x, y, V_{x}, V_{y}, V_{z}\right) d V_{z}, \\
& g_{1}\left(x, y, V_{x}, V_{y}, t\right)=\int_{\mathbb{R}} V_{z}^{2} f_{0}\left(x, y, V_{x}, V_{y}, V_{z}\right) d V_{z} \\
& g_{2}\left(x, y, V_{x}, V_{y}, t\right)=\int_{\mathbb{R}} f_{1}\left(x, y, V_{x}, V_{y}, V_{z}\right) d V_{z},
\end{aligned}
$$

the modelling equations (2.15) can be transformed and integrated with respect to $V_{z}$ with weighting factors 1 and $V_{z}^{2}$ of the following forms,

$$
\frac{\partial g_{i}}{\partial t}+V_{x} \frac{\partial g_{i}}{\partial x}+V_{y} \frac{\partial g_{i}}{\partial y}=v\left(G_{i}^{E S}-g_{i}\right), \quad i=0,1,2
$$

with

$$
\begin{aligned}
& G_{0}^{E S}=\frac{n}{\pi \sqrt{\left(\mathcal{T}_{x x} \mathcal{T}_{y y}-\mathcal{T}_{x y}^{2}\right)}} \exp \left[-\frac{\left(V_{x}-U\right)^{2} \mathcal{T}_{y y}-2\left(V_{x}-U\right)\left(V_{y}-V\right) \mathcal{T}_{x y}+\left(V_{y}-V\right)^{2} \mathcal{T}_{y y}}{\mathcal{T}_{x x} \mathcal{T}_{y y}-\mathcal{T}_{x y}^{2}}\right], \\
& G_{1}^{E S}=\frac{\mathcal{T}_{z z}}{2} G_{0}^{E S}, \quad G_{2}^{E S}=\frac{\delta T_{r e l}}{2} G_{0}^{E S}, \\
& \boldsymbol{\Theta}_{x x}=\frac{2}{n} \int_{\mathbb{R}^{2}} V_{x}^{2} g_{0}\left(x, y, V_{x}, V_{y}, t\right) d V_{x} d V_{y}-2 U^{2}, \\
& \boldsymbol{\Theta}_{y y}=\frac{2}{n} \int_{\mathbb{R}^{2}} V_{y}^{2} g_{0}\left(x, y, V_{x}, V_{y}, t\right) d V_{x} d V_{y}-2 V^{2}, \\
& \boldsymbol{\Theta}_{z z}=\frac{2}{n} \int_{\mathbb{R}^{2}} g_{1}\left(x, y, V_{x}, V_{y}, t\right) d V_{x} d V_{y}, \\
& \boldsymbol{\Theta}_{x y}=\boldsymbol{\Theta}_{y x}=\frac{2}{n} \int_{\mathbb{R}^{2}} V_{x} V_{y} g_{0}\left(x, y, V_{x}, V_{y}, t\right) d V_{x} d V_{y}-2 U V, \\
& \mathcal{T}_{j k}=(1-\theta)\left[(1-\beta) T_{t r} \Delta_{j k}+\beta \boldsymbol{\Theta}_{j k}\right]+\theta T_{o v} \Delta_{j k}, \quad(j, k=x, y, z) .
\end{aligned}
$$


Correspondingly, the macroscopic flow variables for the two dimensional gas dynamics can be obtained by the following expressions,

$$
\begin{aligned}
& n(x, y, t)=\int_{\mathbb{R}^{2}} g_{0} d V_{x} d V_{y,} \\
& U_{i}(x, y, t)=\frac{1}{n} \int_{\mathbb{R}^{2}} V_{i} g_{0} d V_{x} d V_{y}, \\
& T_{t r}(x, y, t)=\frac{2}{3 n} \int_{\mathbb{R}^{2}}\left[\left(V_{x}^{2}+V_{y}^{2}\right) g_{0}+g_{1}\right] d V_{x} d V_{y}-\frac{2}{3}\left(U^{2}+V^{2}\right), \\
& T_{r o t}(x, y, t)=\frac{2}{\delta n} \int_{\mathbb{R}^{2}} g_{2} d V_{x} d V_{y}, \quad T_{o v}(x, y, t)=\frac{3 T_{t r}+\delta T_{r o t}}{3+\delta}, \\
& T_{r e l}(x, y, t)=\theta T_{o v}+(1-\theta) T_{r o t}, \\
& P(x, y, t)=\varsigma \cdot n T_{o v}, \quad \tau_{i j}(x, y, t)=-\varsigma \cdot n \Theta_{i j}+P \Delta_{i j}, \\
& q_{t r, i}(x, y, t)=\int_{\mathbb{R}^{2}} V_{i}\left[\left(V_{x}^{2}+V_{y}^{2}\right) g_{0}+g_{1}\right] d V_{x} d V_{y}-2 U_{i} \int_{\mathbb{R}^{2}} V_{i}^{2} g_{0} d V_{x} d V_{y} \\
& \quad-2 U_{j} \int_{\mathbb{R}^{2}} V_{i} V_{j} g_{0} d V_{x} d V_{y}+n U_{i}\left(U^{2}+V^{2}-\frac{3}{2} T_{t r}\right), \\
& q_{r o t, i}(x, y, t)=\int_{\mathbb{R}^{2}} V_{i} g_{2} d V_{x} d V_{y}-\frac{\delta}{2} n U_{i} T_{r o t}, \\
& q_{i}(x, y, t)=q_{t r, i}+q_{r o t, i} .
\end{aligned}
$$

\section{Numerical procedures of GKUA}

In this section, the numerical procedures of GKUA for the governing equations (2.15) will be presented in detail, including the constructions and applications of the conservative discrete velocity ordinate method (CDVOM) and the lower-upper symmetric GaussSeidel (LU-SGS) implicit scheme, and the computational modeling of the gas-kinetic boundary conditions.

\subsection{Conservative Discrete velocity ordinate method}

The discrete velocity ordinate method (DVOM) in the gas kinetic theory has been presented to remove the VDFs' continuous dependency on the velocity space. Here, we use Eq. (2.31), i.e., the two-dimensional flow, as an example. Using the DVOM in the $V_{x}$ and $V_{y}$ velocity spaces, Eq. (2.31) can be transformed into hyperbolic conservation forms at each of the discrete velocity ordinate points $V_{x \sigma}, V_{y \zeta}\left(\sigma=1,2, \ldots, N_{x \sigma} ; \zeta=1,2, \ldots, N_{y \zeta}\right)$,

$$
\frac{\partial \mathcal{Q}}{\partial t}+\frac{\partial \mathcal{F}}{\partial x}+\frac{\partial \mathcal{G}}{\partial y}=\mathcal{S}
$$


with

$$
\mathcal{Q}=\left(\begin{array}{l}
g_{0 \sigma, \zeta}(x, y, t) \\
g_{1 \sigma, \zeta}(x, y, t) \\
g_{2 \sigma, \zeta}(x, y, t)
\end{array}\right), \quad \mathcal{F}=V_{x \sigma} \mathcal{Q}, \quad \mathcal{G}=V_{y \zeta} \mathcal{Q}, \quad \mathcal{S}=-v \mathcal{Q}+v\left(\begin{array}{l}
G_{0 \sigma, \zeta}^{E S}(x, y, t) \\
G_{1 \sigma, \zeta}^{E S}(x, y, t) \\
G_{2 \sigma, \zeta}^{E S}(x, y, t)
\end{array}\right) .
$$

Here $g_{i \sigma, \zeta}(x, y, t)$ and $G_{i \sigma, \zeta}^{E S}(x, y, t)$ correspond to the values of $g_{i}$ and $G_{i}^{E S}$ at the discrete velocity ordinate points $\left(V_{x \sigma}, V_{y \zeta}\right)$, and the subscript $\sigma$ and $\zeta$ represent the discrete velocity ordinate grid indexes in the $V_{x}$ - and $V_{y}$-direction, respectively.

When the discrete distribution functions $g_{i \sigma, \zeta}(x, y, t)$ are obtained by solving Eq. (3.1), the considered macroscopic flow variables at any time in any position of the physical space can be updated by the appropriate quadrature rules. For example, the gas density by Eq. (2.32a) can be calculated by one numerical quadrature rule as follows,

$$
n(x, y, t)=\sum_{\sigma=1}^{N_{x \sigma}} \sum_{\zeta=1}^{N_{y \zeta}} A_{\sigma} A_{\zeta} \cdot g_{0}\left(x, y, t ; V_{x \sigma}, V_{y \zeta}\right) .
$$

Here the coefficients $A_{\sigma}, A_{\zeta}, V_{x \sigma}$ and $V_{y \zeta}$ are the weights and discrete velocity ordinates, respectively, which are decided by the quadrature rules.

In the past decades, various quadrature rules have been adopted in the GKUA for vast flow simulations successfully, including the Gauss-Hermite, the composite NewtonCotes, the multi-subinterval Gauss-Legendre and the Gauss-Chebyshev quadrature rules. The specific values of the weights and discrete ordinates, and the comparisons of different quadrature rules using in the GKUA can be found in our previous paper [31]. From the comparison, it has been found that the Gauss-Hermite integral methods are the best options for simulating low Mach number flows, which use the least nodes to obtain results with enough computed precision. And the Gauss-Chebyshev rule is actually the most appropriate integration method for some high Mach number complex flows covering various flow regimes. When one rule obtain enough computed precision, it is no need to increase the DVO number or enlarge the discrete velocity space.

However, the equilibrium state $G_{i}^{E S}$ are constructed by the macroscopic flow variables, i.e., $\overrightarrow{\mathbf{W}}=\left(n, U, V, T_{t r}, T_{i n t}, \boldsymbol{\Theta}_{i j}\right)^{T}$, which are calculated by using the numerical quadrature rules, so that the collision conservative constraints (2.22) might not satisfy, leading to a numerical source term. Generally, the conservative method, whose treatments can vanish the numerical source term, maintains the conservation property of the collision integral on a given molecular velocity grid, and do not produce non-physical sources of mass, momentum and energy and therefore in the limit of small Knudsen numbers the kinetic solution approaches the solution of macroscopic flow equations away from boundaries [44]. For the non-conservative method, it will need very fine molecular velocity meshes to keep the numerical source term small when the Knudsen number decreases. Hence, we develop a corresponding CDVOM here, enlightened by Ref. [44]. 
Recalling the conservative condition (2.22), the numerical source term can be written as,

$$
\varepsilon=\sum_{\sigma=1}^{N_{x \sigma}} \sum_{\zeta=1}^{N_{y \zeta}} A_{\sigma} A_{\zeta} \cdot \Psi \cdot\left(\begin{array}{c}
G_{0}^{E S}-g_{0} \\
G_{1}^{E S}-g_{1} \\
G_{2}^{E S}-g_{2}
\end{array}\right)
$$

where

$$
\Psi=\left(\begin{array}{ccc}
1 & 0 & 0 \\
V_{x} & 0 & 0 \\
V_{y} & 0 & 0 \\
V_{x}^{2}+V_{y}^{2} & 1 & 1
\end{array}\right)
$$

Considering the effects of the opposite of the stress tensor $\Theta$ in equilibrium state $G_{i}^{E S}$, we can introduce

$$
\mathrm{Y}=\left(\begin{array}{ccc}
V_{x}^{2} & 0 & 0 \\
V_{x} V_{y} & 0 & 0 \\
V_{y}^{2} & 0 & 0 \\
0 & 1 & 0
\end{array}\right)
$$

and have the following relations,

$$
\int_{\mathbb{R}^{2}} \mathbf{Y}\left(\begin{array}{l}
g_{0} \\
g_{1} \\
g_{2}
\end{array}\right) d V_{x} d V_{y}=\left(\begin{array}{c}
n\left(\frac{1}{2} \mathbf{\Theta}_{x x}+U^{2}\right) \\
n\left(\frac{1}{2} \mathbf{\Theta}_{x y}+U V\right) \\
n\left(\frac{1}{2} \mathbf{\Theta}_{y y}+V^{2}\right) \\
\frac{n}{2} \mathbf{\Theta}_{z z}
\end{array}\right),
$$

and

$$
\int_{\mathbb{R}^{2}} \mathrm{Y}\left(\begin{array}{c}
G_{0}^{E S} \\
G_{1}^{E S} \\
G_{2}^{E S}
\end{array}\right) d V_{x} d V_{y}=\left(\begin{array}{c}
n\left(\frac{1}{2} \mathcal{T}_{x x}+U^{2}\right) \\
n\left(\frac{1}{2} \mathcal{T}_{x y}+U V\right) \\
n\left(\frac{1}{2} \mathcal{T}_{y y}+V^{2}\right) \\
\frac{n}{2} \mathcal{T}_{z z}
\end{array}\right)
$$

Hence, applying the quadrature rule on Eqs. (3.4) and (3.5), and coupling with Eq. (3.3), one can obtain the following new numerical source term,

$$
H=\sum_{\sigma=1}^{N_{x \sigma}} \sum_{\zeta=1}^{N_{y \zeta}} A_{\sigma} A_{\zeta} \cdot\left(\begin{array}{c}
\Psi \\
\mathrm{Y}
\end{array}\right) \cdot\left(\begin{array}{c}
G_{0}^{E S}-g_{0} \\
G_{1}^{E S}-g_{1} \\
G_{2}^{E S}-g_{2}
\end{array}\right)-\mathcal{R},
$$

where

$$
\mathcal{R}=\left(0,0,0,0, \mathcal{T}_{x x}-\boldsymbol{\Theta}_{x x}, \mathcal{T}_{x y}-\boldsymbol{\Theta}_{x y}, \mathcal{T}_{y y}-\boldsymbol{\Theta}_{y y}, \mathcal{T}_{z z}-\boldsymbol{\Theta}_{z z}\right)^{T}
$$

In order to preserve the conservative properties, one should minimize the expression (3.6) into zero, which can be solved by using the Newton-Raphson method for nonlinear system equations with multi-variables. The iterative formula can be expressed as the following form,

$$
M\left(\overrightarrow{\mathbf{W}}^{s}\right)\left(\overrightarrow{\mathbf{W}}^{s+1}-\overrightarrow{\mathbf{W}}^{s}\right)=-H\left(\overrightarrow{\mathbf{W}}^{s}\right) \Rightarrow \overrightarrow{\mathbf{W}}^{s+1}=\overrightarrow{\mathbf{W}}^{s}-M^{-1}\left(\overrightarrow{\mathbf{W}}^{s}\right) H\left(\overrightarrow{\mathbf{W}}^{s}\right),
$$


where the matrix $M=\partial H / \partial W$ is the Jacobian matrix, the subscript $s$ denotes the $s$-th iterative step.

However, due to the complex expressions of $M$ that contains the differential and numerical integration calculations, we can give the approximate $\mathcal{M}_{(*)}$ by integrating the expression first and then differentiating, as well as its inverse matrix $\mathcal{M}_{(*)}^{-1}$,

$$
\begin{aligned}
& \mathcal{M}_{(*)}=\left(\begin{array}{cccccccc}
1 & 0 & 0 & 0 & 0 & 0 & 0 & 0 \\
U & n & 0 & 0 & 0 & 0 & 0 & 0 \\
V & 0 & n & 0 & 0 & 0 & 0 & 0 \\
\frac{\delta}{2} T_{i n t}+\frac{3}{2} T_{t r}+U^{2}+V^{2} & 2 n U & 2 n V & \frac{3 n}{2} & \frac{\delta n}{2} & 0 & 0 & 0 \\
\frac{1}{2} \mathbf{\Theta}_{x x}+U^{2} & 2 n U & 0 & 0 & \frac{n}{2} & 0 & 0 & 0 \\
\frac{1}{2} \Theta_{x y}+U V & n V & n U & 0 & 0 & \frac{n}{2} & 0 & 0 \\
\frac{1}{2} \Theta_{y y}+V^{2} & 0 & 2 n V & 0 & 0 & 0 & \frac{n}{2} & 0 \\
\frac{1}{2} \Theta_{z z} & 0 & 0 & 0 & 0 & 0 & 0 & \frac{n}{2}
\end{array}\right), \\
& \mathcal{M}_{(*)}^{-1}=\frac{1}{n}\left(\begin{array}{cccccccc}
n & 0 & 0 & 0 & 0 & 0 & 0 & 0 \\
-U & 1 & 0 & 0 & 0 & 0 & 0 & 0 \\
-V & 0 & 1 & 0 & 0 & 0 & 0 & 0 \\
\frac{1}{3}\left[\delta \boldsymbol{\Theta}_{x x}-\delta T_{i n t}-3 T_{t r}-2(\delta-1) U^{2}+2 V^{2}\right] & \frac{4}{3}(\delta-1) U & -\frac{4}{3} V & \frac{2}{3} & -\frac{2}{3} \delta & 0 & 0 & 0 \\
2 U^{2}-\boldsymbol{\Theta}_{x x} & -4 U & 0 & 0 & 2 & 0 & 0 & 0 \\
-\boldsymbol{\Theta}_{x y}+4 U V-2 \mathrm{UV} & -2 V & -2 U & 0 & 0 & 2 & 0 & 0 \\
2 V^{2}-\boldsymbol{\Theta}_{y y} & 0 & -4 V & 0 & 0 & 0 & 2 & 0 \\
-\boldsymbol{\Theta}_{z z} & 0 & 0 & 0 & 0 & 0 & 0 & 2
\end{array}\right) .
\end{aligned}
$$

\subsection{Explicit and implicit schemes}

Based on the cell-centered finite volume method (FVM), the Runge-Kutta explicit and LU-SGS implicit scheme is constructed to solve the discrete hyperbolic conservation equation (3.1) directly.

At one discrete velocity ordinate point $\left(V_{x \sigma}, V_{y \zeta}\right)$, for one control volume $\Omega_{I, J}$ of grid central-type element (see Fig. 1), the integral type of Eq. (3.1) is of the following form,

$$
\frac{\partial \mathcal{Q}_{I, J}}{\partial t}+\frac{1}{\Omega_{I, J}} \oint_{\partial \Omega} \overrightarrow{\mathcal{F}} \cdot \vec{n} d s=\mathcal{S}_{I, J}
$$

with

$$
\overrightarrow{\mathcal{F}}=\left(V_{x \sigma} \vec{i}+V_{y \zeta} \vec{j}\right) \mathcal{Q}_{I, J}, \quad \mathcal{S}_{I, J}=-v \mathcal{Q}_{I, J}+v\left(G_{0}^{E S}, G_{1}^{E S}, G_{2}^{E S}\right)_{I, J^{\prime}}^{T}
$$

where $\vec{n}$ is the normal vector on boundaries of the control volume, and $\mathcal{Q}_{I, J}$ is the average value of $\mathcal{Q}$ in $\Omega_{I, J}$.

The second term in the left hand side of Eq. (3.9) can be rewritten as

$$
\frac{1}{\Omega_{I, J}} \oint_{\partial \Omega} \overrightarrow{\mathcal{F}} \cdot \vec{n} d s=\frac{1}{\Omega_{I, J}}\left(\mathcal{H}_{I+\frac{1}{2}, J}-\mathcal{H}_{I-\frac{1}{2}, J}+\mathcal{H}_{I, J+\frac{1}{2}}-\mathcal{H}_{I, J-\frac{1}{2}}\right),
$$




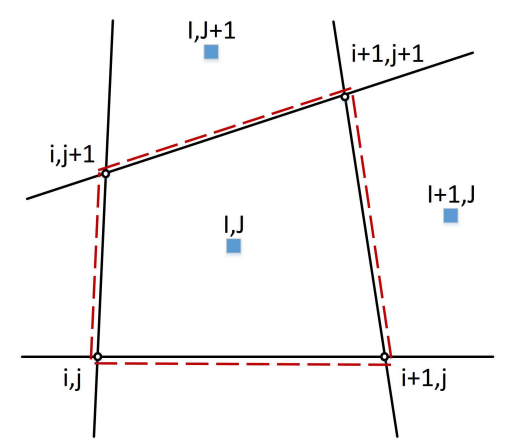

Figure 1: Control volume $\Omega_{I, J}$ of grid central-type element.

with

$$
\mathcal{A}_{I+\frac{1}{2}, J}=\left[\left(V_{x \sigma} n_{x}+V_{y \zeta} n_{y}\right) \Delta s\right]_{I+\frac{1}{2}, J^{\prime}} \quad \mathcal{H}_{I+\frac{1}{2}, J}=\mathcal{A}_{I+\frac{1}{2}, J} \mathcal{Q}_{I+\frac{1}{2}, J} .
$$

Applying the Steger-Warming flux vector splitting (FVS) method, the fluxes at the interface of the control volume can be split as positive and negative fluxes, which can be expressed of the follows,

$$
\mathcal{H}_{I+\frac{1}{2}, J}=\mathcal{H}_{I+\frac{1}{2}, J}^{+}+\mathcal{H}_{I+\frac{1}{2}, J}^{-}=\mathcal{A}_{I+\frac{1}{2}, J}^{+} \mathcal{Q}_{I+\frac{1}{2}, J}^{L}+\mathcal{A}_{I+\frac{1}{2}, J}^{-} \mathcal{Q}_{I+\frac{1}{2}, J^{\prime}}^{R}
$$

where

$$
\mathcal{A}_{I+\frac{1}{2}, J}^{ \pm}=\frac{1}{2}\left(\mathcal{A}_{I+\frac{1}{2}, J} \pm\left|\mathcal{A}_{I+\frac{1}{2}, J}\right|\right) .
$$

Here, we can use the reconstruction method to obtain the values of $\mathcal{Q}_{I+\frac{1}{2}, J}^{L}$ and $\mathcal{Q}_{I+\frac{1}{2}, J}^{R}$. For example, using the classical 2nd order MUSCL implementation with minmod limiter [45], one can obtain

$$
\begin{aligned}
& \mathcal{Q}_{I+\frac{1}{2}, J}^{L}=\mathcal{Q}_{I, J}+\left(X_{I+\frac{1}{2}}-X_{I}\right) \operatorname{minmod}\left(\frac{\mathcal{Q}_{I+1, J}-\mathcal{Q}_{I, J}}{X_{I+1}-X_{I}}, \frac{\mathcal{Q}_{I, J}-\mathcal{Q}_{I-1, J}}{X_{I+1}-X_{I}}\right), \\
& \mathcal{Q}_{I+\frac{1}{2}, J}^{R}=\mathcal{Q}_{I+1, J}-\left(X_{I+1}-X_{I+\frac{1}{2}}\right) \min \bmod \left(\frac{\mathcal{Q}_{I+2, J}-\mathcal{Q}_{I+1, J}}{X_{I+2}-X_{I+1}}, \frac{\mathcal{Q}_{I+1, J}-\mathcal{Q}_{I, J}}{X_{I+1}-X_{I}}\right),
\end{aligned}
$$

where $X$ represents the coordinate, and

$$
\min \bmod (a, b)=\frac{\operatorname{sgn}(a)+\operatorname{sgn}(b)}{2} \cdot \min (|a|,|b|), \quad \operatorname{sgn}(a)= \begin{cases}1, & a>0 \\ 0, & a=0 \\ -1, & a<0\end{cases}
$$

The controlling equation (3.9) can rewritten as the following form,

$$
\frac{\partial \mathcal{Q}_{1, J}}{\partial t}=R H S
$$


with

$$
R H S=-\frac{1}{\Omega_{I, J}}\left(\mathcal{H}_{I+\frac{1}{2}, J}-\mathcal{H}_{I-\frac{1}{2}, J}+\mathcal{H}_{I, J+\frac{1}{2}}-\mathcal{H}_{I, J-\frac{1}{2}}\right)+\mathcal{S}_{I, J}
$$

As an explicit temporal scheme, the improved Euler, or second-order Runge-Kutta, method can be utilized to solve the above equation and update the values of VDF at this discrete velocity ordinate point $\left(V_{x \sigma}, V_{y \zeta}\right)$ for all physical cell,

$$
\begin{aligned}
& \mathcal{Q}_{I, J}^{*}=\mathcal{Q}_{I, J}^{(n)}+d t \cdot \operatorname{RHS}\left(\mathcal{Q}_{I, J}^{(n)}\right), \\
& \mathcal{Q}_{I, J}^{(n+1)}=\frac{1}{2}\left[\mathcal{Q}_{I, J}^{(n)}+\mathcal{Q}_{I, J}^{*}+d t \cdot \operatorname{RHS}\left(\mathcal{Q}_{I, J}^{*}\right)\right] .
\end{aligned}
$$

For the explicit time step, according to Ref. [46], one can obtain

$$
\Delta t=C F L \cdot \min \left[\frac{\Omega_{I, J}}{\max _{\sigma, \zeta}\left(\Lambda^{I}+\Lambda^{J}\right)_{I, J}}\right]
$$

where the subscript $(I, J)$ represents the value at the $(I, J)$-th grid cell, and $\Lambda$ is the spectral radii of the convective flux Jacobians, which has the following form,

$$
\Lambda^{I}=\frac{1}{2}\left\{\mathcal{A}_{I-\frac{1}{2}, J}+\mathcal{A}_{I+\frac{1}{2}, J}\right\}, \quad \Lambda^{I}=\frac{1}{2}\left\{\mathcal{A}_{I, J-\frac{1}{2}}+\mathcal{A}_{I, J+\frac{1}{2}}\right\}
$$

where the CFL number is the adjusting coefficient determined by the complexity of the problem and the change of computational residual, which is set to 0.9 generally. The discretized velocity domain becomes to be larger for hypersonic complex flows, the time step should be controlled in the actual computation.

In order to improve the computational efficiency, we can construct the implicit temporal scheme based on the LU-SGS, as the following procedures. At $t^{(n+1)}$ time level, the controlling equation (3.9) becomes

$$
\frac{\partial \mathcal{Q}_{I, J}^{(n+1)}}{\partial t}+\frac{1}{\Omega_{I, J}} \oint_{\partial \Omega} \overrightarrow{\mathcal{F}}(n+1) \cdot \vec{n} d s-\mathcal{S}_{I, J}^{(n+1)}=0 .
$$

Adding $\operatorname{RHS}\left(\mathcal{Q}_{I, J}^{(n)}\right)$ at the both sides of the above equation, one can obtain

$$
\frac{\partial \mathcal{Q}_{I, J}^{(n+1)}}{\partial t}+\frac{1}{\Omega_{I, J}} \oint_{\partial \Omega}\left(\overrightarrow{\mathcal{F}}^{(n+1)}-\overrightarrow{\mathcal{F}}^{(n)}\right) \cdot \vec{n} d s-\left(\mathcal{S}_{I, J}^{(n+1)}-\mathcal{S}_{I, J}^{(n)}\right)=R H S_{I, J}^{(n)}
$$

For the solution of stationary flow problems, the first-order accurate in time is applied since it requires less computer memory,

$$
\frac{\Delta \mathcal{Q}_{I, J}}{\Delta t}+\frac{1}{\Omega_{I, J}} \oint_{\partial \Omega} A_{n} \Delta \mathcal{Q}_{I, J} d s-A_{v} \Delta \mathcal{Q}_{I, J}=R H S_{I, J}^{(n)}
$$


where $\Delta \mathcal{Q}_{I, J}=\mathcal{Q}_{I, J}^{(n+1)}-\mathcal{Q}_{I, J}^{(n)}, A_{n}=A \cdot \vec{n}, A=\partial \overrightarrow{\mathcal{F}} / \partial \mathcal{Q}=\left(V_{x \sigma} \vec{i}+V_{y \zeta} \vec{j}\right)$, and $A_{v}=-v(1-$ $\left.\partial \mathcal{Q}^{E S} / \partial \mathcal{Q}\right)$. According to the first-order accurate in time [47], the local equilibrium VDF can be seen as constant between these two time level, leading the approximate expression $A_{v} \approx-v$.

Similarly, using the FVS method, one can obtain

$$
\begin{aligned}
& \oint_{\partial \Omega} A_{n} \Delta \mathcal{Q}_{I, J} d s=\oint_{\partial \Omega}\left(A_{n}^{+} \Delta \mathcal{Q}_{I, J}+A_{n}^{-} \Delta \mathcal{Q}_{I, J}\right) d s \\
= & \left(\mathcal{A}_{I+\frac{1}{2}, J}^{+} \Delta \mathcal{Q}_{I, J}+\mathcal{A}_{I+\frac{1}{2}, J}^{-} \Delta \mathcal{Q}_{I+1, J}\right)-\left(\mathcal{A}_{I-\frac{1}{2}, J}^{+} \Delta \mathcal{Q}_{I-1, J}+\mathcal{A}_{I-\frac{1}{2}, J}^{-} \Delta \mathcal{Q}_{I, J}\right) \\
& +\left(\mathcal{A}_{I, J+\frac{1}{2}}^{+} \Delta \mathcal{Q}_{I, J}+\mathcal{A}_{I, J+\frac{1}{2}}^{-} \Delta \mathcal{Q}_{I, J+1}\right)-\left(\mathcal{A}_{I, J-\frac{1}{2}}^{+} \Delta \mathcal{Q}_{I, J-1}+\mathcal{A}_{I, J-\frac{1}{2}}^{-} \Delta \mathcal{Q}_{I, J}\right) .
\end{aligned}
$$

If defining the following terms,

$$
\begin{aligned}
& \alpha_{I, J}=\Omega_{I, J}\left(\frac{1}{\Delta t}+v\right)+\mathcal{A}_{I+\frac{1}{2}, J}^{+}-\mathcal{A}_{I-\frac{1}{2}, J}^{-}+\mathcal{A}_{I, J+\frac{1}{2}}^{+}-\mathcal{A}_{I, J-\frac{1}{2}{ }^{\prime}}^{-} \\
& \alpha_{I+1, J}=\mathcal{A}_{I+\frac{1}{2} J^{\prime}}^{-} \quad \alpha_{I, J+1}=\mathcal{A}_{I, J+\frac{1}{2}}^{-} \\
& \alpha_{I-1, J}=-\mathcal{A}_{I-\frac{1}{2}, J^{\prime}}^{+} \quad \alpha_{I, J-1}=-\mathcal{A}_{I, J-\frac{1}{2}}^{+} \quad b_{I, J}=\Omega_{I, J} R H S_{I, J}^{(n)},
\end{aligned}
$$

and assuming the node numbers at the $X$ - and $Y$-directions are $N_{x}$ and $N_{y}$ respectively, Eq. (3.17) can be rewritten of the following form,

$$
M \Delta \mathcal{Q}=\mathbf{b},
$$

where

$$
\begin{aligned}
& \Delta \mathcal{Q}=\left(\Delta \mathcal{Q}_{1,1}, \Delta \mathcal{Q}_{1,2}, \cdots, \Delta \mathcal{Q}_{1, N_{y}}, \Delta \mathcal{Q}_{2,1}, \cdots, \Delta \mathcal{Q}_{N_{x}, N_{y}}\right)^{T}, \\
& \mathbf{b}=\left(b_{1,1}, b_{1,2}, \cdots, b_{1, N_{y}}, b_{2,1}, \cdots, b_{N_{x}, N_{y}}\right)^{T}, \\
& M_{k, l}= \begin{cases}\alpha_{I, J}, & k=l=(I-1) * N_{y}+J, \\
\alpha_{I+1, J}, & k=(I-1) * N_{y}+J, l=I * N_{y}+J, \\
\alpha_{I, J+1}, & k=(I-1) * N_{y}+J, l=(I-1) * N_{y}+J+1, \\
\alpha_{I-1, J}, & k=(I-1) * N_{y}+J, l=(I-2) * N_{y}+J, \\
\alpha_{I, J-1}, & k=(I-1) * N_{y}+J, l=(I-1) * N_{y}+J-1, \\
0, & \text { otherwise, }\end{cases} \\
& \left(1 \leqslant k, l \leqslant N_{x} * N_{y}\right) .
\end{aligned}
$$

The above equation is a five-diagonal linear equation system, which can be solved by the approximate LU decomposition method. Introducing the decomposition $M=D+L+U$, where the matrix $D$ is the diagonal of $M, L$ its strict lower part, and $U$ its strict upper part, we have the following approximate expression,

$$
\mathbf{b}=(D+L+U) \Delta \mathcal{Q} \approx(D+L) D^{-1}(D+U) \Delta \mathcal{Q} .
$$


Hence, Eq. (3.20) can be solved by two steps,

$$
\begin{aligned}
& (D+L) \cdot \boldsymbol{\Delta} \mathcal{Q}^{*}=\mathbf{b}, \\
& (D+U) \cdot \Delta \mathcal{Q}=D \Delta \mathcal{Q}^{*} .
\end{aligned}
$$

In conclusion, the numerical procedures of the implicit LU-SGS scheme for Eq. (3.9) can be expressed as follows:

Step 1. Calculating the right side term $R H S_{I, J}^{(n)}$ by Eq. (3.13).

Step 2. Scanning upwards. From Eq. (3.22a), one can obtain

$$
\begin{gathered}
\alpha_{I, J} \Delta \mathcal{Q}_{I, J}^{*}-\mathcal{A}_{I-\frac{1}{2}, J}^{+} \Delta \mathcal{Q}_{I-1, J}^{*}-\mathcal{A}_{I, J-\frac{1}{2}}^{+} \Delta \mathcal{Q}_{I, J-1}^{*}=\Omega_{I, J} R H S_{I, J}^{(n)} \\
\Rightarrow \Delta \mathcal{Q}_{I, J}^{*}=\left(\Omega_{I, J} R H S_{I, J}^{(n)}+\mathcal{A}_{I-\frac{1}{2}, J}^{+} \Delta \mathcal{Q}_{I-1, J}^{*}+\mathcal{A}_{I, J-\frac{1}{2}}^{+} \Delta \mathcal{Q}_{I, J-1}^{*}\right) / \alpha_{I, J} .
\end{gathered}
$$

Step 3. Scanning downwards. From Eq. (3.22b), one can obtain

$$
\begin{gathered}
\alpha_{I, J} \Delta \mathcal{Q}_{I, J}+\mathcal{A}_{I+\frac{1}{2}, J}^{-} \Delta \mathcal{Q}_{I+1, J}+\mathcal{A}_{I, J+\frac{1}{2}}^{-} \Delta \mathcal{Q}_{I, J+1}=\alpha_{I, J} \Delta \mathcal{Q}_{I, J}^{*} \\
\Rightarrow \Delta \mathcal{Q}_{I, J}=\Delta \mathcal{Q}_{I, J}^{*}-\left(\mathcal{A}_{I+\frac{1}{2}, J}^{-} \Delta \mathcal{Q}_{I+1, J}+\mathcal{A}_{I, J+\frac{1}{2}}^{-} \Delta \mathcal{Q}_{I, J+1}\right) / \alpha_{I, J} .
\end{gathered}
$$

Step 4. Advancing in time and updating VDF at this discrete velocity ordinate point $\left(V_{x \sigma}, V_{y \delta}\right)$,

$$
\mathcal{Q}_{I, J}^{(n+1)}=\mathcal{Q}_{I, J}^{(n)}+\Delta \mathcal{Q}_{I, J}
$$

The time step $\Delta t$ in the implicit scheme is less strict than that of the explicit schemes. Hence, for the implicit scheme, the CFL number can be set as 0.9 initially and equably increased to one set value with the time step growing to a certain number.

In addition, one flag of the program end is whether the value of the residual error is lower than the given accuracy $\varepsilon_{0}$ or not. We use the root-mean-square deviation of the macroscopic flow variables as the residual error, which is defined as follows,

$$
\varepsilon=\frac{1}{\Delta t} \sqrt{\frac{1}{M_{x} M_{y} M_{z}} \sum_{i=1}^{M_{x}} \sum_{j=1}^{M_{y}} \sum_{k=1}^{M_{z}}\left(\frac{E^{(n+1)}-E^{(n)}}{E^{(n)}}\right)^{2}},
$$

where $E$ represents one macroscopic flow variable, $M_{x}, M_{y}$ and $M_{z}$ are the node numbers of the three physical dimensions, respectively, and the superscripts $(n)$ and $(n+1)$ denote the two time levels. Generally, we use the density, and set $\varepsilon_{0}=10^{-5}$.

Furthermore, the initialization procedures are the beginning of the numerical simulations, which are important to reduce unnecessary memory waste and guarantee the processes running smoothly. As same as our previous works, we initialize the molecular VDFs $f_{0}$ and $f_{1}$ with the equilibrium distribution functions $f_{0}^{E S}$ and $f_{1}^{E S}$, respectively. 
Generally, the flow variables are initializing by the given properties in the whole flow field.

However, one inevitable problem occurred in this procedure is the unknown value of the corrected tensor $\mathcal{T}$, which is the moment of the VDFs over the molecular velocity space, like the density, flow velocity and temperature, while do not have an unambiguous physical meanings comparing with other flow properties.

The concrete initial value of the tensor $\mathcal{T}$ can be calculated by some flow variables, which can be proofed in the following derivation processes. For the conciseness of our derivation procedures given in this paper, we take the two-dimensional flow as an example. Firstly, the VDFs are initialized with their equilibrium states,

$$
\begin{aligned}
& g_{0}=G_{0}^{E S}=\frac{n}{\pi \sqrt{\left(\mathcal{T}_{x x} \mathcal{T}_{y y}-\mathcal{T}_{x y}^{2}\right)}} \exp \left[-\frac{\left(V_{x}-u\right)^{2} \mathcal{T}_{y y}-2\left(V_{x}-u\right)\left(V_{y}-v\right) \mathcal{T}_{x y}+\left(V_{y}-v\right)^{2} \mathcal{T}_{y y}}{\mathcal{T}_{x x} \mathcal{T}_{y y}-\mathcal{T}_{x y}^{2}}\right], \\
& g_{1}=G_{1}^{E S}=\frac{\mathcal{T}_{z z}}{2} g_{0}, \quad g_{2}=G_{2}^{E S}=\frac{\delta T_{r e l}}{2} g_{0} .
\end{aligned}
$$

With the following notations,

$$
D=\mathcal{T}_{x x} \mathcal{T}_{y y}-\mathcal{T}_{x y}^{2}, \quad p=\frac{D}{\mathcal{T}_{y y}}, \quad q=\frac{D}{\mathcal{T}_{x x}}, \quad r=\frac{D}{2 \mathcal{T}_{x y}},
$$

the expression of $g_{0}$ can be rewritten as

$$
g_{0}=\frac{n}{\pi \sqrt{D}} \exp \left[-\frac{\left(V_{x}-u\right)^{2}}{p}-\frac{\left(V_{y}-v\right)^{2}}{q}+\frac{\left(V_{x}-u\right)\left(V_{y}-v\right)}{r}\right] .
$$

For the definition of the tensor $\Theta$, it can be calculated as

$$
\begin{aligned}
& \boldsymbol{\Theta}_{x x}=\frac{2}{n} \int_{\mathbb{R}^{2}}\left(V_{x}-u\right)^{2} g_{0} d V_{x} d V_{y}=\frac{2}{n} \cdot \frac{n}{\pi \sqrt{D}} \cdot 4 \pi \sqrt{q} r^{3}\left(\frac{p}{4 r^{2}-p q}\right)^{3 / 2}=\mathcal{T}_{x x}, \\
& \boldsymbol{\Theta}_{y y}=\frac{2}{n} \int_{\mathbb{R}^{2}}\left(V_{y}-v\right)^{2} g_{0} d V_{x} d V_{y}=\frac{2}{n} \cdot \frac{n}{\pi \sqrt{D}} \cdot 4 \pi \sqrt{p} r^{3}\left(\frac{q}{4 r^{2}-p q}\right)^{3 / 2}=\mathcal{T}_{y y}, \\
& \boldsymbol{\Theta}_{x y}=\boldsymbol{\Theta}_{y x}=\frac{2}{n} \int_{\mathbb{R}^{2}}\left(V_{x}-u\right)\left(V_{y}-v\right) g_{0} d V_{x} d V_{y}=\frac{2}{n} \cdot \frac{n}{\pi \sqrt{D}} \cdot 2 \pi r^{2}\left(\frac{p q}{4 r^{2}-p q}\right)^{3 / 2}=\mathcal{T}_{x y}, \\
& \boldsymbol{\Theta}_{z z}=\frac{2}{n} \int_{\mathbb{R}^{2}} f_{1} d V_{x} d V_{y}=\frac{2}{n} \cdot \frac{\mathcal{T}_{z z} n}{2}=\mathcal{T}_{z z} .
\end{aligned}
$$

As all four type temperatures have been set as $T_{0}$, i.e., $T_{t r}=T_{r o t}=T_{o v}=T_{r e l}=T_{0}$, and recalling the definition of $\mathcal{T}$ below Eq. (2.31), one can obtain

$$
\mathcal{T}=\left(\begin{array}{ccc}
T_{0} & 0 & 0 \\
0 & T_{0} & 0 \\
0 & 0 & T_{0}
\end{array}\right)
$$

The same conclusion can be deduced in the one- and three-dimensional flows, which are omitted here for the sake of the conciseness of this paper. 


\subsection{Boundary condition procedures}

Instead of using the macroscopic flow variables, the boundary conditions of the GKUA are numerically implemented on the VDFs directly, as our algorithm focuses on the time evolution of the molecular VDFs explicitly at each points in the physical and velocity space. Without loss of generality, we use the two-dimensional flow case as an example, and the situations of the three-dimensional flow can be extended by following the derivation processes.

\subsubsection{Solid wall boundary}

For the solid wall boundary, it is under the assumption that the gas molecules striking the surface are subsequently emitted with the equilibrium VDF partly accommodating to the velocity $\vec{U}_{w}=\left(U_{w}, V_{w}\right)$ and wall temperature $T_{w}$, coupling with the specular reflection, which is characterized by the accommodation coefficient $\eta$. In practical terms, the procedures can be described as follows:

Define $C_{n}=\overrightarrow{\mathbf{C}} \cdot \overrightarrow{\mathbf{n}}$, where $\overrightarrow{\mathbf{n}}$ is the outward unit vector normal to the wall surface. When $C_{n}>0$ in the wall cells, for the equilibrium VDF fully accommodation boundary, the reflected VDFs from the wall surface can be written as the equilibrium states. From the discussions in the last part, we can find that the corrected tensor $\mathcal{T}=T_{0} \cdot \mathbf{I}_{\mathbf{d}}$ at the equilibrium state. Hence, one can obtain that $\mathcal{T}_{x x}=\mathcal{T}_{y y}=\mathcal{T}_{z z}=T_{w}$ and $\mathcal{T}_{x y}=0$ for the reflected VDFs. The expression of the reflected VDFs can be simplified as

$$
g_{0, e}^{+}=\frac{n_{w}}{\pi T_{w}} \exp \left[-\frac{\left(V_{x}-u_{w}\right)^{2}+\left(V_{y}-v_{w}\right)^{2}}{T_{w}}\right], \quad g_{1, e}^{+}=\frac{T_{w}}{2} g_{0, w^{\prime}}^{+} \quad g_{2, e}^{+}=\delta g_{1, w^{\prime}}^{+}
$$

where $n_{w}$ is the number density of the diffused molecules. Considering the conservation of the mass flux normal to the wall surface, which means that the incident molecules equals the reflected ones at any time,

$$
\int_{C_{n}>0} C_{n} \cdot g_{0, e}^{+} d V_{x} d V_{y}+\int_{C_{n}<0} C_{n} \cdot g_{0, e}^{-} d V_{x} d V_{y}=0,
$$

one can obtain

$$
n_{w}=-\sqrt{\frac{\pi}{T_{w}}} \int_{\mathbb{R}^{2}}\left(C_{n}-\left|C_{n}\right|\right) g_{0, e}^{-} d V_{x} d V_{y} .
$$

Once the number density of molecules diffused from the solid surface $n_{w}$ has been calculated, the reflected VDFs can be obtained by Eq. (3.32).

For the specular reflection wall boundary, it has the expression

$$
g_{i, s}(\overrightarrow{\mathbf{C}})=g_{i}(\overrightarrow{\mathbf{C}}-2(\overrightarrow{\mathbf{n}} \cdot \overrightarrow{\mathbf{C}}) \overrightarrow{\mathbf{n}}) .
$$

And the reflected velocity distribution functions can be obtained by

$$
g_{i, w}=\eta g_{i, e}+(1-\eta) g_{i, s} .
$$


We set $\eta=1$ at the computations in the remaining part of this article, if it has not been mentioned specifically.

When $C_{n} \leqslant 0$, the discrete VDFs at the wall cells can be interpolated by the secondorder upwind-difference method with the values at the adjacent grids.

\subsubsection{Inlet, outlet and far-field boundaries}

The VDFs for outgoing molecules through the inlet or outlet boundary are numerically interpolated by the second-order difference approximation according to the upwind nature of the interior point scheme. For incoming molecules from outside, the VDFs are set as an equilibrium distribution with prescribed free stream properties when it is along upstream boundary ahead of the body. And the VDFs do not have gradient along the outward direction in the downstream boundary.

For the far-field boundary, we utilize the characteristics conditions to treat the VDFs at the boundary.

\subsubsection{Periodic boundary}

For the periodic boundary condition, it assumes that the entrance and exit boundaries are connected, which means the molecular velocity distribution functions of the outside fictitious nodes are copied from the functions of the interior nodes, which is illustrated in Fig. 2.

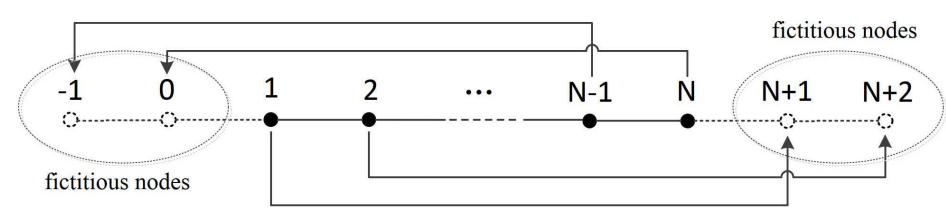

Figure 2: Schematic diagram of the periodic boundary condition. The arrow lines represent the replication processes.

\subsubsection{Symmetrical boundary}

For the symmetrical boundary condition, the VDF $f^{r}$ in the fictitious cells can be expressed by that of the interior flow field,

$$
f^{r}(\overrightarrow{\mathbf{r}}, \overrightarrow{\mathbf{V}}, t)=f(\overrightarrow{\mathbf{r}}-2(\overrightarrow{\mathbf{n}} \cdot \overrightarrow{\mathbf{r}}) \overrightarrow{\mathbf{n}}, \overrightarrow{\mathbf{V}}-2(\overrightarrow{\mathbf{n}} \cdot \overrightarrow{\mathbf{v}}) \overrightarrow{\mathbf{n}}, t),
$$

where $\overrightarrow{\mathbf{n}}$ is the unit vector normal to the symmetrical line and pointing to the interior of the flow field.

In addition, the flow chart of the GKUA with implicit FVM scheme is shown in Fig. 3. The right upper dashed box represents the selection process of a suitable quadrature rule 


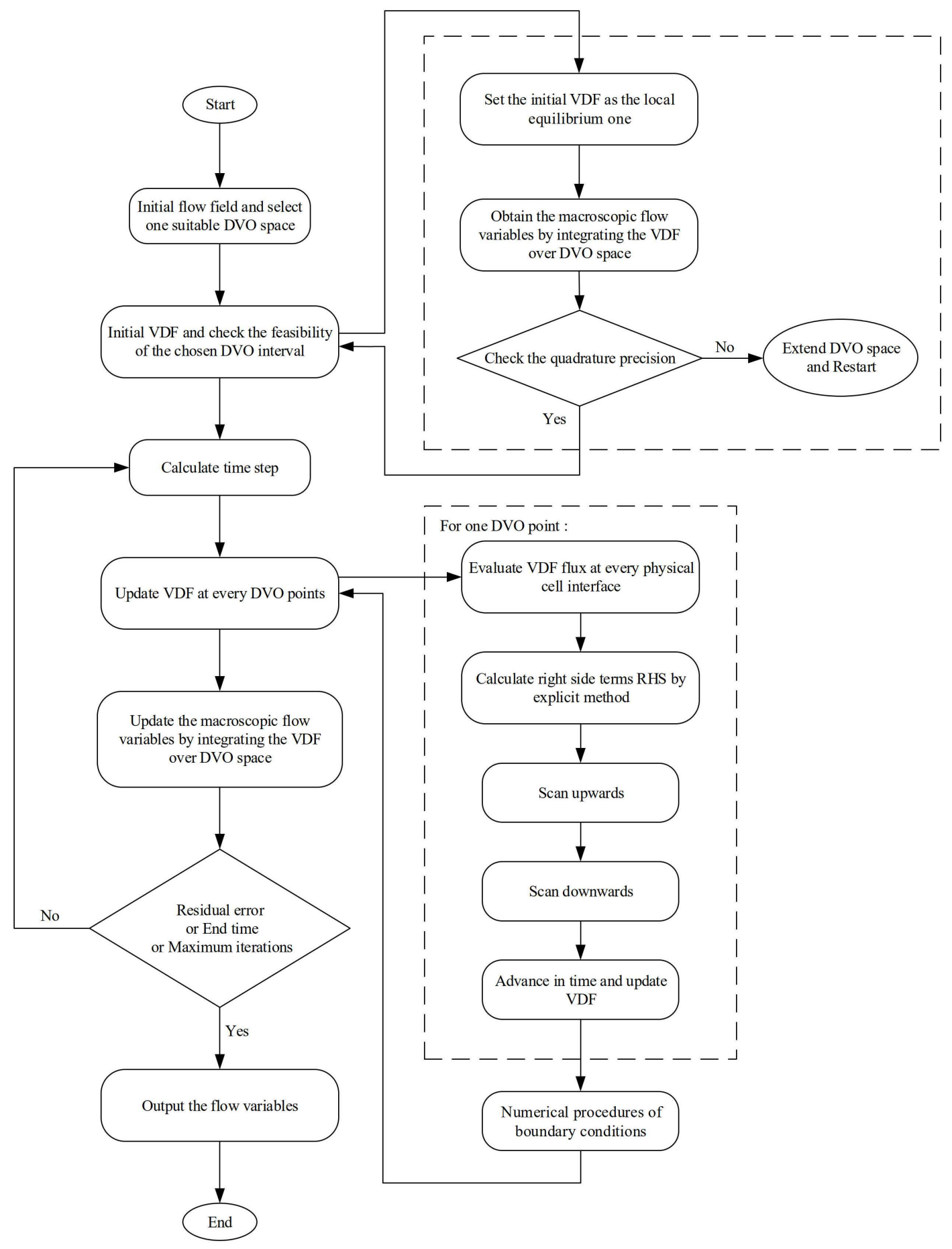

Figure 3: Flowchart of the GKUA. 
for the current simulation. We estimate the maximum values of the flow velocity and temperature in the flow-field, set an initial DVO interval by the " $3 \sigma^{\prime \prime}$ principle, obtain the macroscopic flow variables by integrating the VDF over DVO space, and check the accuracy. If the accuracy is enough, the initial procedure of the GKUA codes is completed. If not, the initial DVO interval should be extended, following the increase of the DVO number, and the previous procedures will be repeated until the enough accuracy is obtained.

It should note that a uniform truncated velocity space is applied at the every physical cell in the flow field, as well as the number of DVO, and Eq. (3.9) is solved at every discrete velocity ordinate point $\left(V_{x \sigma}, V_{y \delta}\right)$.

\section{Numerical simulations and discussion}

In this section, some selected computational examples, including the Sod Riemann problem in broad range of Knudsen numbers, the rotational relaxation in a homogeneous gas, the normal shock structure calculations, the planar Fourier and Couette flows, the flow around a circular cylinder and the hypersonic flow pasting a re-entry plate placed normally, are solved and analyzed. These examples can be used to validate the feasibility and accuracy of the new version GKUA for the polyatomic gas flow simulations in rotational non-equilibrium state, covering various flow regimes. All numerical simulations are conducted on a personal computer with Intel(R) Core(TM) i7-7700 CPU @ $3.6 \mathrm{GHz}$ and single thread.

\subsection{Sod Riemann problem}

The one-dimensional Riemann problem can be described by the way where a diaphragm located at $x=0.5$ divides a one-dimensional flow field into two regions, each in a constant equilibrium state at $t=0$. Here, we investigate the Sod Riemann problem with the monatomic and diatomic gases, respectively, in order to test the compatibility of our algorithm for polyatomic gases presented in this paper.

The initial conditions for the Sod Riemann problem [48] are given as,

$$
(\rho, P, U)= \begin{cases}(1.0,1.0,0.0) & \text { when } 0.0 \leqslant x \leqslant 0.5 \\ (0.125,0.1,0.0) & \text { when } 0.5<x \leqslant 1.0\end{cases}
$$

The simulation is performed on a uniform mesh with $N=1001$ until final time $t=0.2$. The time step is determined by Eq. (3.15), and the CFL number is set as 0.9.

Firstly, in order to compare the CDVOM and DVOM in the present algorithm, the composite Simpson quadrature rule has been applied with different number of the DVO nodes. In this comparison, we use the $A r$ gas at $K n=10^{-4}$, whose parameters are set as $\gamma=5 / 3, \operatorname{Pr}=2 / 3$, and $\delta=10^{-5}$. Three cases of DVO nodes, i.e., 61, 27, and 21, are uniformly distributed in an interval $[-7.5,7.5]$, whose uniform spacing $\Delta V_{x}=0.25,0.5769$, 


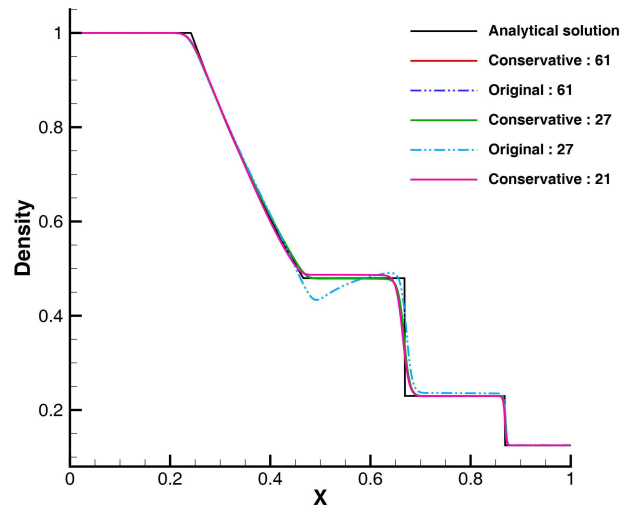

(a) Flow velocity

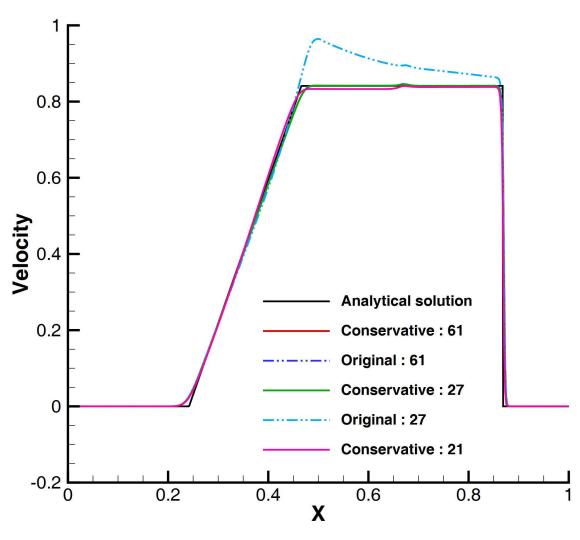

(b) Temperature

Figure 4: Comparisons between the original and conservative DVOM of the Sod shock-tube problem using monatomic gas.

and 0.75 , respectively. Fig. 4 presents the computed density and velocity profiles by using the DVOM and CDVOM, as well as the analytical solutions. It can be found that the numerical simulation results with enough integral nodes match with the analytical solutions well. And the conservative method can obtain the same accurate results with fewer nodes than that of the original one. Following the increase of $\Delta V_{x}$, the simulation profiles gradually deviate. The CDVOM results are more accurate than the DVOM results at the same node. For the case of $N=21$, the results of CDVOM almost agree with the cases with more nodes, while the original one is diverging. This phenomenon is caused by that the velocity grids are not fine enough to accurately evaluate the moment integrals of the distribution function for flow variables and the compatibility condition of the discrete collision integral is not well preserved by the nonconservative calculations of collision integral. To sum up, the present CDVOM are better than the original method in the aspect of the storage space and whole computational time, which is utilized at the remaining computational results of this paper if it is not mentioned especially.

For the diatomic gas simulations, we choose the nitrogen $\left(N_{2}\right)$ gas, where the ratio of specific heats $\gamma=1.4$ and $\operatorname{Pr}=0.72$. Other parameters are set as $\alpha=1, \omega=0.5, \beta=-0.5$ and $\theta=1 / 3$. The modified Gauss-Hermite quadrature rule with 32 discrete velocity ordinate points is applied for the simulations to evaluate the macroscopic flow moments over the velocity space, and the reference temperature is set as $T_{r e f}=273 \mathrm{~K}$. Fig. 5 presents the density, flow velocity, temperature and pressure profiles obtained by the present GKUA at different Knudsen numbers, as well as the Riemann theoretic result that solve the Euler equations in continuum flow regime. It can be observed that the numerical simulation results of $K n=10^{-5}$ agree with the theoretical solutions well, which shows the accuracy and feasibility of the present algorithm in solving the continuum diatomic gas flows. 


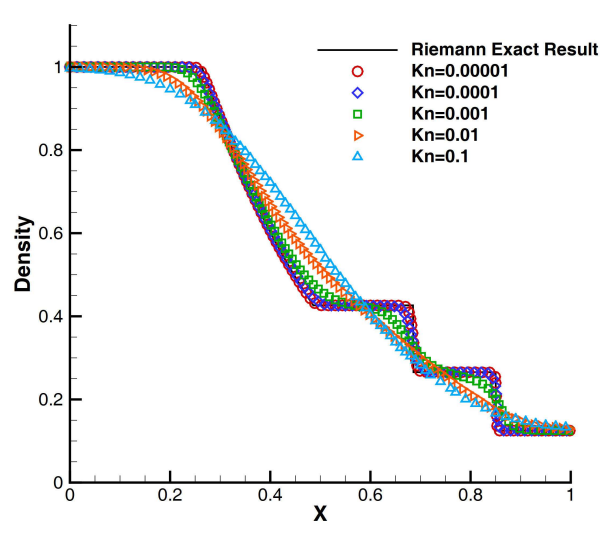

(a) Density

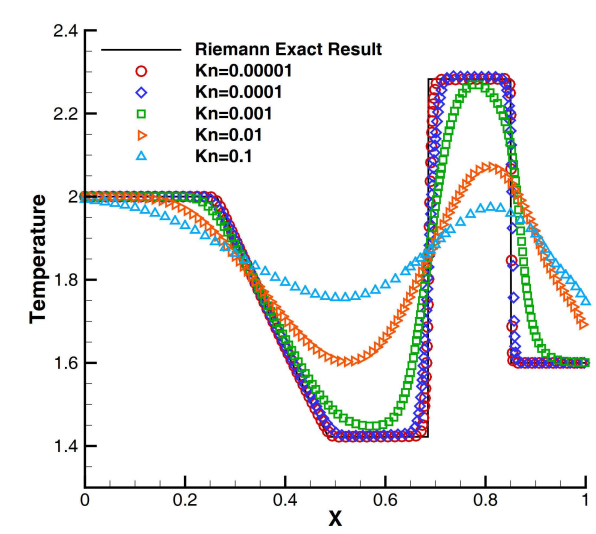

(c) Temperature

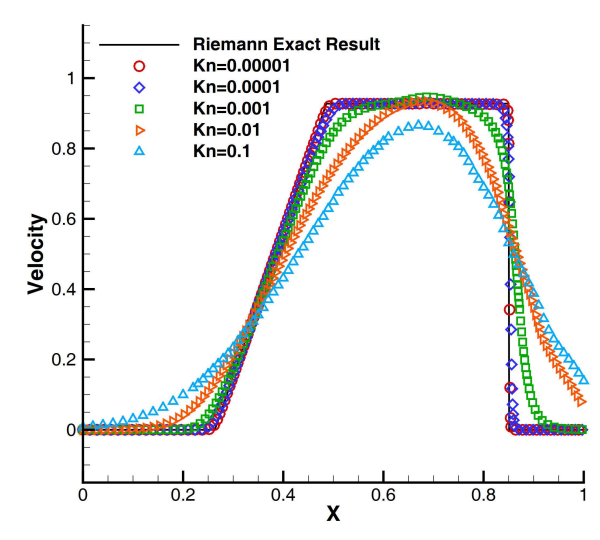

(b) Flow velocity

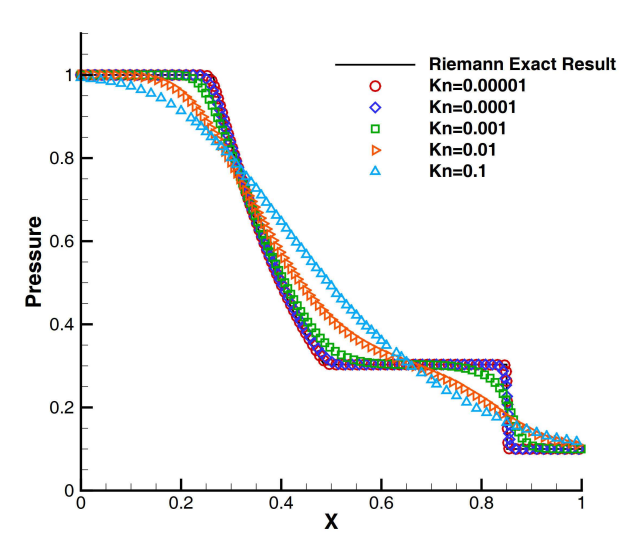

(d) Pressure

Figure 5: Macroscopic flow variable profiles of the Sod Riemann problem at time $t=0.2$ for the $N_{2}$ gas.

Besides, we can find that when the Knudsen number becomes bigger, i.e., from $K n=10^{-5}$ to $K n=0.01$, the thicker is the shock and expansion wave. For the rarefied flow of $K n=0.1$, there only exists strong disturbance without the shock or expanding wave. It can be indicated from the varying distributions in Fig. 5 that the gas flow intuitively change with the multi-scale characteristics covering various flow regimes from the continuous flow of $K n=10^{-5}$ to the rarefied flow of $K n=0.1$.

In addition, an interesting phenomenon is shown in Fig. 6, which gives the profiles of the translational temperature $T_{t r}$, the rotational temperature $T_{r o t}$ and the overall temperature $T_{e q}$ under different Knudsen numbers. It can be observed that the distributions of $T_{t r}$ and $T_{r o t}$ agree with the profile of $T_{e q}$ when the value of Knudsen number is small, representing the continuum flow regime, while differences among these three profiles of 


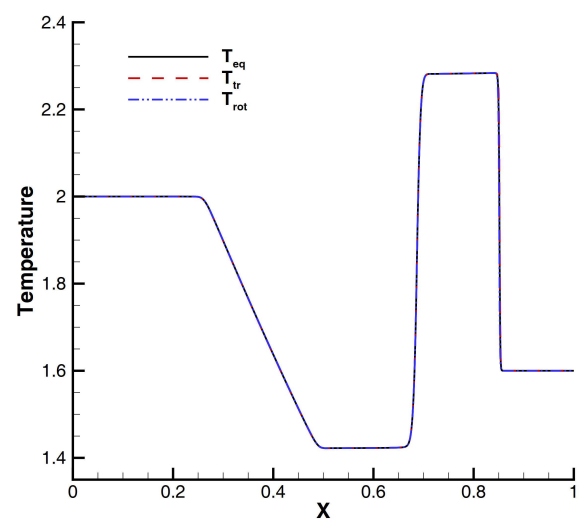

(a) $K n=10^{-5}$

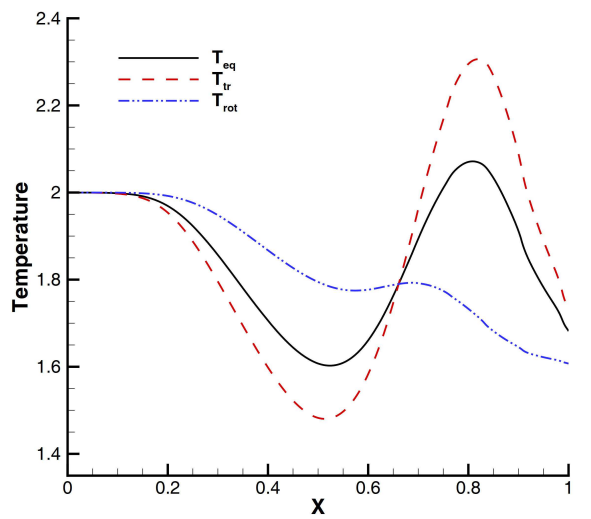

(c) $K n=0.01$

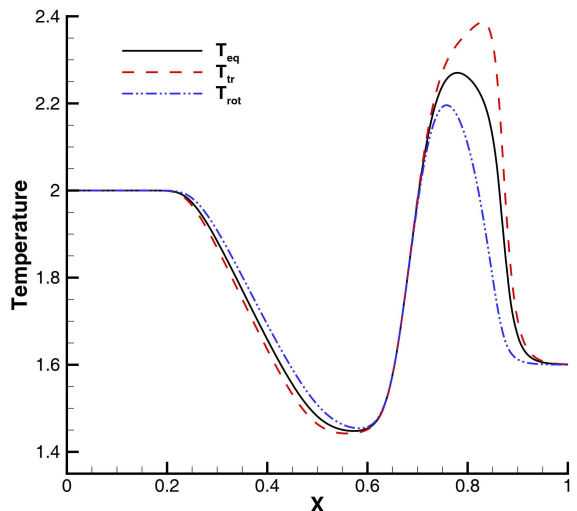

(b) $K n=0.001$

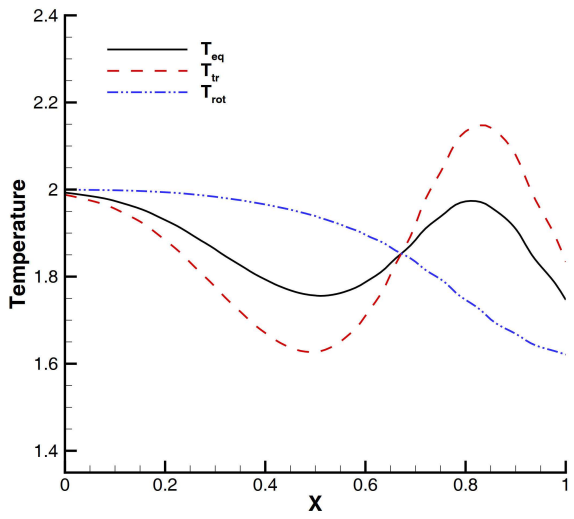

(d) $K n=0.1$

Figure 6: Profiles of the three type temperatures $T_{t r}, T_{r o t}$ and $T_{o v}$ under different $K n$ at time $t=0.2$.

temperature occur when the flow regime changes from the continuum to the rarefied. In the transition flow, e.g., $K n=0.001$ and $K n=0.01$, the these three profile tendencies of temperature are the same at the expanding wave part, and have deviation at the shock transition zone. While in the rarefied flow, i.e., $K n=0.1$, the distinctions among $T_{t r}, T_{\text {rot }}$ and $T_{e q}$ are quite obvious, even the profile tendencies of the translational and internal temperatures become different, reflecting the strong non-equilibrium effects in the rarefied gas flows.

Furthermore, the comparisons of the flow variables between the monatomic and diatomic gases for the Sod Riemann problem in the continuum regime are presented in Fig. 7. Here, the modified Gauss-Hermite quadrature rule with 48 DVO points is applied for the monatomic gas simulation, while the diatomic case uses 32 DVO points, due to the 


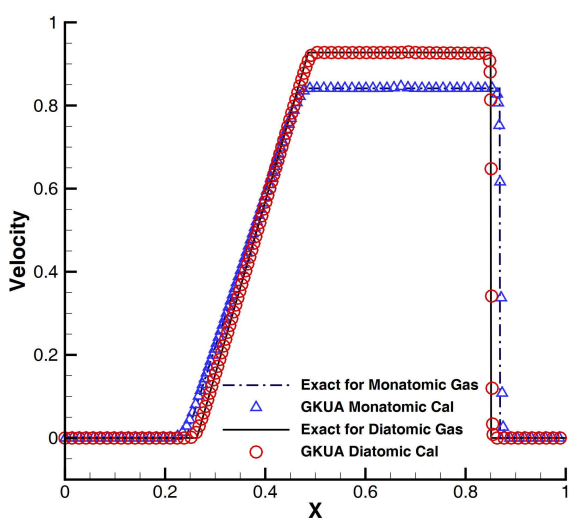

(a) Flow velocity

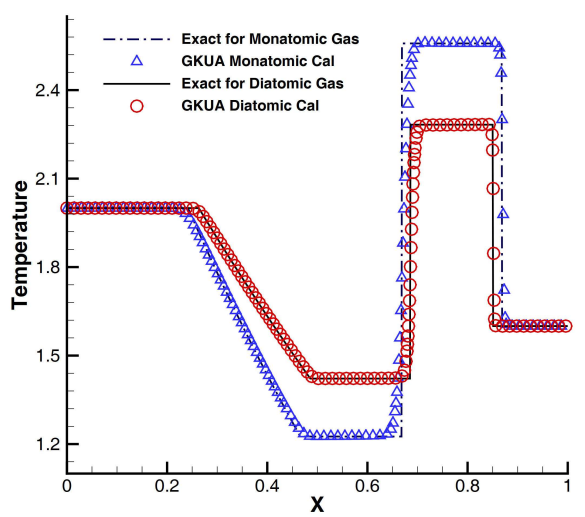

(b) Temperature

Figure 7: Comparisons of the flow variables between the monatomic and diatomic gases of the Sod shock-tube problem at time $t=0.2$.

different needed integral interval. It can be found that the gaseous medium and the ratio of specific heats have impact on the flow structure and the profiles of the macroscopic variables.

\subsection{Rotational relaxation in a homogeneous gas}

In the case with constant mean collision frequency $v$ and rotational collision number $Z$, leading to constant relaxation parameter $\theta$, the analytical solutions of Eq. (2.27) can be obtained as the following forms,

$$
\left\{\begin{array}{l}
T_{t r}=\frac{3 T_{t r}(0)+\delta T_{r o t}(0)+\delta e^{-\theta v t}\left[T_{t r}(0)-T_{r o t}(0)\right]}{3+\delta} \\
T_{r o t}=\frac{3 T_{t r}(0)+\delta T_{r o t}(0)-3 e^{-\theta v t}\left[T_{t r}(0)-T_{r o t}(0)\right]}{3+\delta}
\end{array}\right.
$$

where $T_{t r}(0)$ and $T_{r o t}(0)$ are the initial values of the translational temperature $T_{t r}$ and rotational temperature $T_{r o t}$, respectively. Recalling the definition of the mean collision frequency (2.13), it can be a constant value only for the Maxwell molecule with $\chi=1$. For other type molecules, e.g., the hard sphere with $\chi=0.5$ and the nitrogen with $\chi=0.72$, there exist no exact analytical solutions.

In order to test the present algorithm for the rotational relaxation procedures in a homogeneous gas, the comparisons between the GKUA results and the analytical solutions (4.2) for different $Z$ are presented in Fig. 8, using the Maxwell molecules. The mean collision time (m.c.t.) is calculated using the overall temperature $T_{o v}$ in all cases to normalize the time. The initial state is set as $T_{t r}(0)=500 \mathrm{~K}, T_{\text {rot }}(0)=0 \mathrm{~K}$, and without lost 


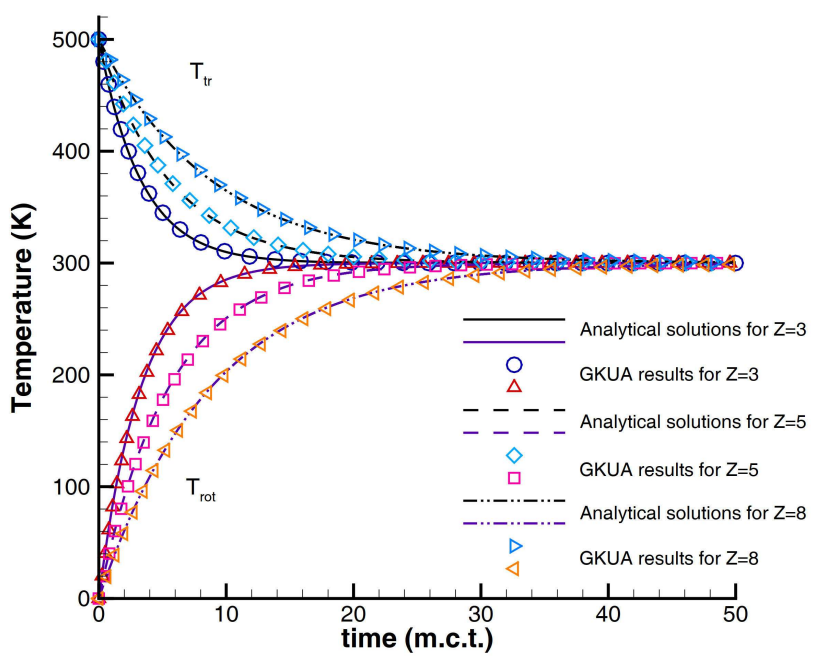

Figure 8: Rotational relaxation in a homogeneous gas.

generality, the non-dimensional number density is $\widetilde{n}=1.0$ and the Knudsen number is fixed at $K n=1.0$. The simulation is performed on a uniform mesh with $N=500$ until final time $t=50.0$. The time step is determined by Eq. (3.15), and the CFL number is set as 0.9. The modified Gauss-Hermite quadrature rule with 32 discrete velocity ordinate points is applied for the simulations to evaluate the macroscopic flow variables over the velocity space, and the reference temperature is set as $T_{r e f}=273 \mathrm{~K}$.

From Fig. 8, it can be observed that the GKUA results match well with the analytical solutions in these three cases, and the value of $Z$ has an impact on the speed of the relaxation to the equilibrium state. With the increase of $Z$, the process slows down, while the final equilibrium states are the same.

\subsection{Normal shock structure calculations}

In the case of the normal shock wave, there is no stream speed in the plane of the wave, and the transition is from a supersonic upstream (state 1) flow to a subsonic downstream (state 2) flow. The shock Mach number $M_{s}$ is defined as the ratio of the wave speed to the sound speed in the upstream gas. The upstream state is chosen as the reference state with $\rho_{1}=1, T_{1}=1$ and $U_{1}=M_{s} \sqrt{\gamma P_{1} / \rho_{1}}$, and the downstream state can be calculated through the Rankine-Hugoniot conditions,

$$
\frac{\rho_{2}}{\rho_{1}}=\frac{U_{2}}{U_{1}}=\frac{M_{s}^{2}}{1+\frac{\gamma-1}{\gamma+1}\left(M_{s}^{2}-1\right)}, \quad \frac{P_{2}}{P_{1}}=\frac{2 \gamma}{\gamma+1} M_{s}^{2}-\frac{\gamma-1}{\gamma+1}, \quad T_{2}=\frac{P_{2}}{\rho_{2}} .
$$


The length scale is normalized by the mean free path $\lambda_{1}$ in front of the shock wave, which is of the following form,

$$
\lambda_{1}=\frac{16}{5} \sqrt{\frac{\gamma}{2 \pi}} \frac{\mu_{1}}{\rho_{1} a_{1}}
$$

where $\mu$ is viscosity, and $a$ is the speed of sound. The flow region is extended over a distance $-x_{1} \leqslant x \leqslant x_{2}$, and the discontinuity between upstream and downstream states is set at $x=0$ as the initial condition. Besides, in order to describe the profiles of the density and temperature, we introduce the following two terms,

$$
d \rho=\frac{\rho-\rho_{1}}{\rho_{2}-\rho_{1}}=\frac{n-n_{1}}{n_{2}-n_{1}}, \quad d T=\frac{T-T_{1}}{T_{2}-T_{1}} .
$$

The simulation is performed on the interval $x \in[-35,35]$, which is divided into a uniform mesh with $N=350$, until the final non-dimensional time $t=200$ or the residual error is less than $10^{-12}$. The time step is determined by Eq. (3.15), and the CFL number is set as 0.9. The composite Newton-Cotes numerical quadrature rule is employed to evaluate the macroscopic flow variables over the velocity space, whose interval is $V_{x} \in$ $[-15,30]$ and discrete with 361 points. The reference temperature is set as $T_{r e f}=273 \mathrm{~K}$, and the Knudsen number is set as $K n=1.0$.

In order to describe the non-equilibrium flow properties in the interior of a normal shock wave and demonstrate the reliability of the present algorithm in solving the shock problems, we use the Argon gas on behalf of the monatomic gas to simulate this problem. The ratio of specific heats $\gamma=5 / 3$ and $P r=2 / 3$. Other parameters are set as $\alpha=1, \omega=0.5$, $\beta=-0.5$ and $\theta=0$, and the excited internal energy levels $\operatorname{DoF} \delta$ is set as $\delta=10^{-5}$.

Fig. 9 shows the measured density and temperature profiles for Mach numbers $M_{s}=$ $1.55,2.05,3.8,9.0$ corresponding to the viscosity-temperature index $\chi=0.8077$ with the comparison of the results of the DSMC and the Mott-Smith moment method [49], respectively. From Fig. 9, it can be observed that the present GKUA solutions of the shock profiles for the low Mach number $M_{s}=1.55$, the intermediate Mach number $M_{s}=2.05,3.8$ are in good agreement with the DSMC results and the Mott-Smith moment method results, while the temperature distribution of the high Mach number $M_{S}=9.0$ has deviation from other two methods, which may be caused by the differences among three computable models. Besides, it can be shown that the Mott-Smith moment method fits well to describe the density distribution for the structure of shock waves, however, it is difficult to give the temperature distribution since temperature is a more sensitive quantity as a higher moment of the velocity distribution function, and the Mott-Smith theory is better for lower that than for higher Mach number flow. It can be confirmed from the present computations in Fig. 9 over the range of Mach numbers $M_{s} \in[1.55,9.0]$ that the density profiles are nearly symmetric on the point of the intermediate density between the upstream and downstream states. While as the shock becomes stronger, a marked asymmetry and overshoot phenomena has arisen in the temperature profiles, which reveals the severe rarefied non-equilibrium effects existing in the strong shock waves. 


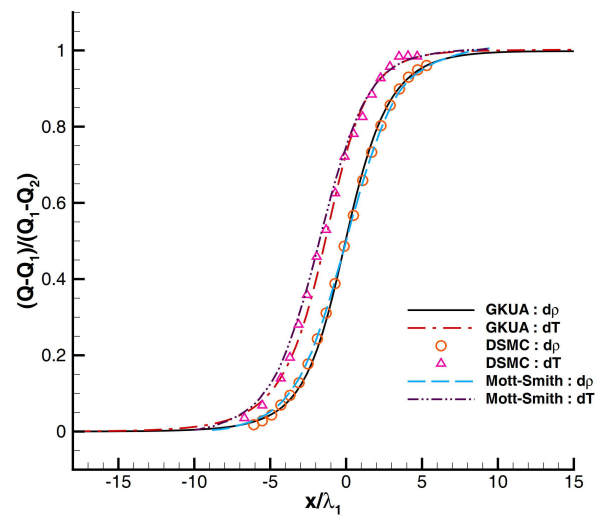

(a) $M_{S}=1.55$

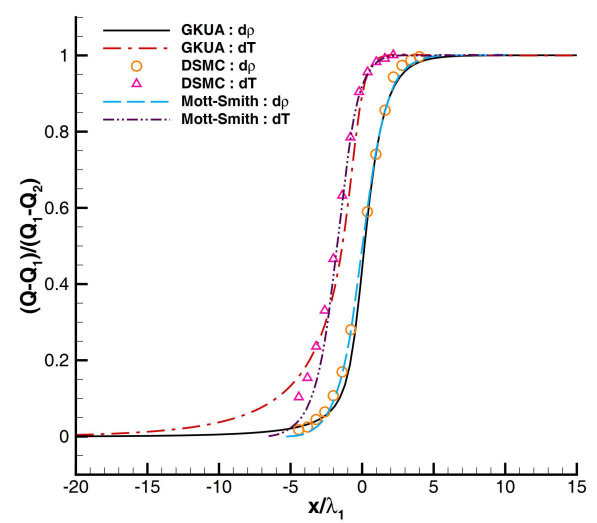

(c) $M_{S}=3.8$

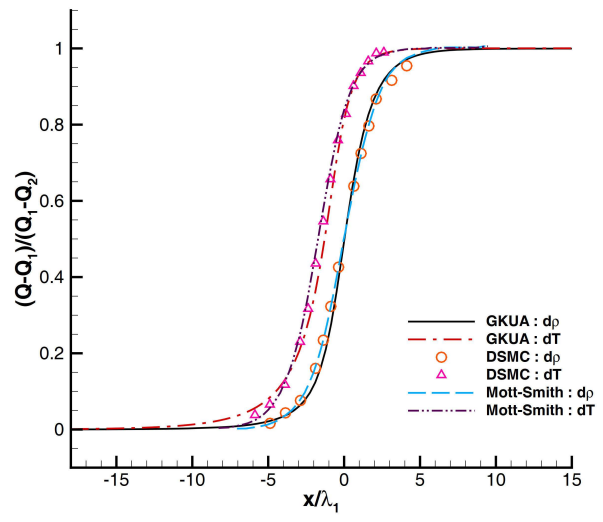

(b) $M_{S}=2.05$

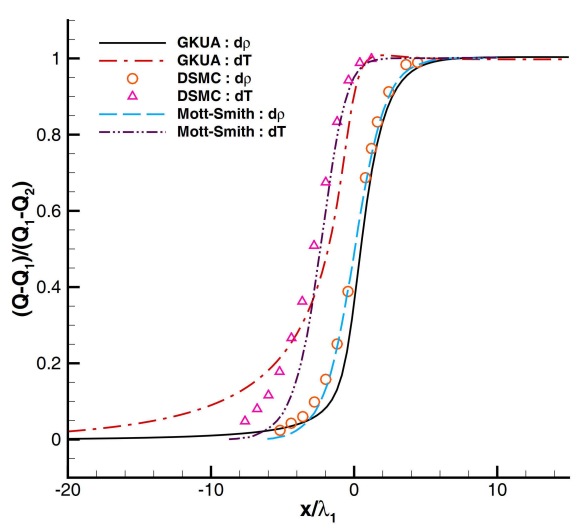

(d) $M_{S}=9.0$

Figure 9: Comparisons of the GKUA, DSMC and Mott-Smith theory results for the normal shock structures at different Mach numbers in Argon gas.

For the diatomic gases, we choose the nitrogen gas, where the viscosity-temperature index $\chi=0.74$. Other parameters are set as $\alpha=1, \omega=0.5$ and $\beta=-0.5$. The parameter $\theta$ is a changing variable in different Mach numbers, for $M_{s}=1.53, \theta=0.333$; for $M_{s}=2.0$, $\theta=0.333$; for $M_{s}=2.8, \theta=0.319$; for $M_{s}=6.1, \theta=0.306$; for $M_{s}=10.0, \theta=0.273$, which are determined by the rotational collision number $Z$ given in Ref. [49].

Fig. 10 shows the density profiles obtained by the present GKUA, experiment and DSMC for different Mach numbers, from which it can be indicated that the GKUA solutions are in agreements with the experiment and DSMC results, which are presented in Ref. [49]. Besides, the translational, rotational and overall temperature profiles obtained by the present GKUA, experiment and DSMC are presented in Fig. 11, which 


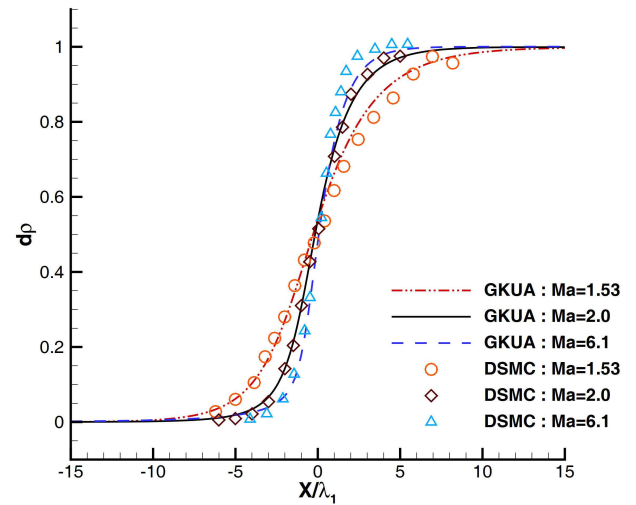

(a)

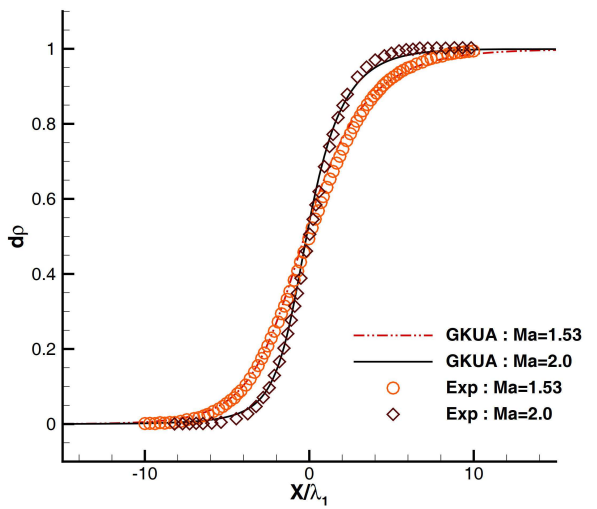

(b)

Figure 10: Density profiles under different Mach numbers for the diatomic gases. (a) GKUA solutions and the DSMC results; (b) GKUA solutions and the experiment results.

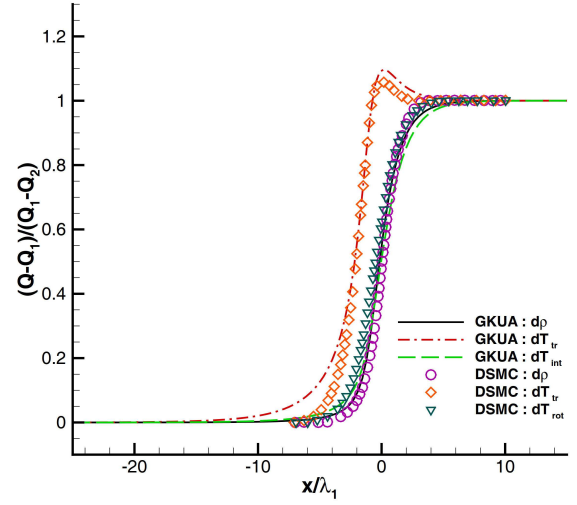

(a) $M_{S}=2.8$

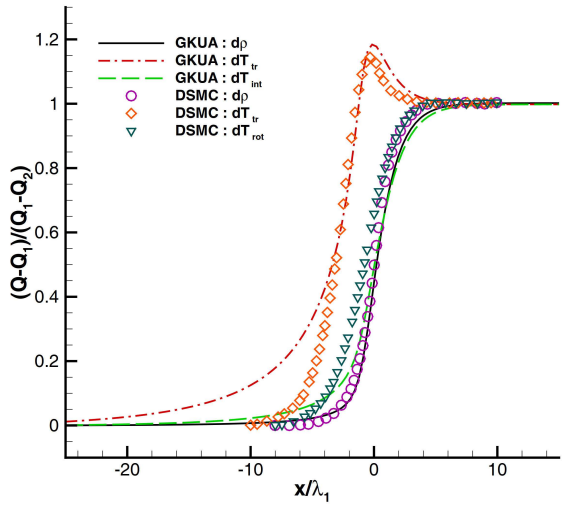

(b) $M_{S}=10.0$

Figure 11: Translational, rotational and overall equilibrium temperature profile comparisons among the GKUA, experiment and DSMC results.

are in agreements with each other. Besides, it can be observed that at high Mach number, the translational temperature in the shock structure goes up earlier in comparison with the DSMC results, which indicates that the wider domain of thermodynamic nonequilibrium including thermal conductivity and viscosity exists in the close upstream for stronger shock wave flow, and the more significant asymmetric distribution exists in the profiles of the translational temperature for higher Mach number. 


\subsection{Planar Fourier and Couette flows}

The planar Fourier and Couette flows are encountered in micro-motors, comb mechanisms, and micro-bearings [28]. For the planar Fourier flows, we consider the nitrogen gas between two parallel plates a distance $L$ apart. The normalized temperature of the lower plate at $Y_{l}=0$ is $T_{l}=2 T_{0} / 3$, while that of the upper plate at $Y_{u}=L$ is $T_{u}=4 T_{0} / 3$. The temperature exponent of the viscosity coefficient is $\chi=0.74$, and other parameters are set as $\alpha=1, \omega=0.5, \beta=-0.5$ and $\theta$ is determined by Eq. (2.29), where the rotational collision number is set as $Z=2.226$.

The simulation is performed on the rectangle domain $[0,1] \times[-0.05,0.05]$, which are meshed with $100 \times 10$, until the residual error is less than $10^{-12}$. The time step is determined by Eq. (3.15), and the CFL number is set as 0.9. The modified Gauss-Hermite integral rule with $24 \times 24 \mathrm{DVO}$ points is applied in this simulation. The diffusive reflection boundary conditions are utilized at the upward and downward plates, while the characteristics-based boundary conditions are used at the inlet and outlet parts of the domain. Two different Knudsen numbers $K n=0.1$ and $K n=1.0$ are tested here.

Fig. 12 shows the resulting density and translational temperature profiles (the rotational temperatures are not shown because they are very close to the translational ones) in this planar Fourier flow. Excellent agreement among the results of the GKUA, model in Ref. [12] and the DSMC simulations can be seen.

The planar Couette flow configuration is the same as for the planar Fourier flow above, although the two plates now have the same temperature $T_{0}$, and the top plate moves in X-direction with a speed $V_{m}$ while the bottom plate moves in the opposite direction at the same speed. In the simplest case, the Couette flow can be used as a prototype flow to model such flows driven by a moving plate. In this simulation, we consider

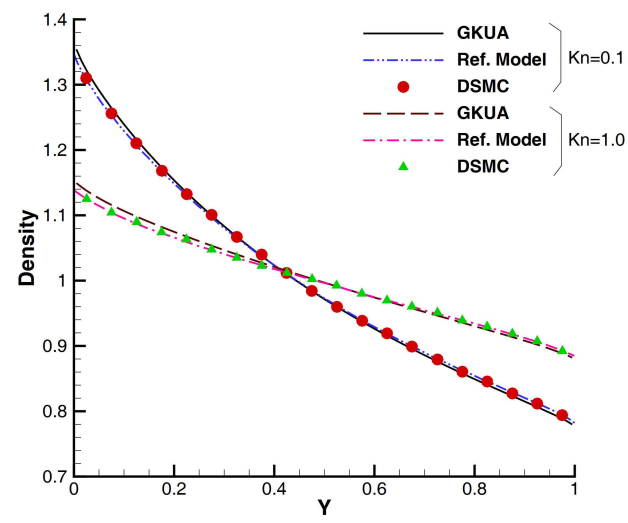

(a) Density

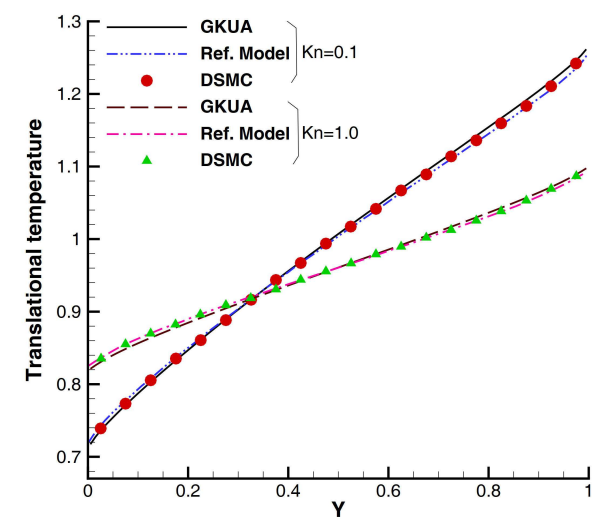

(b) Translational temperature

Figure 12: Comparisons of the normalized variables between the GKUA, model in Ref. [12] and DSMC simulations for planar Fourier flows with nitrogen gases at $K n=0.1$ and $K n=1.0$. 


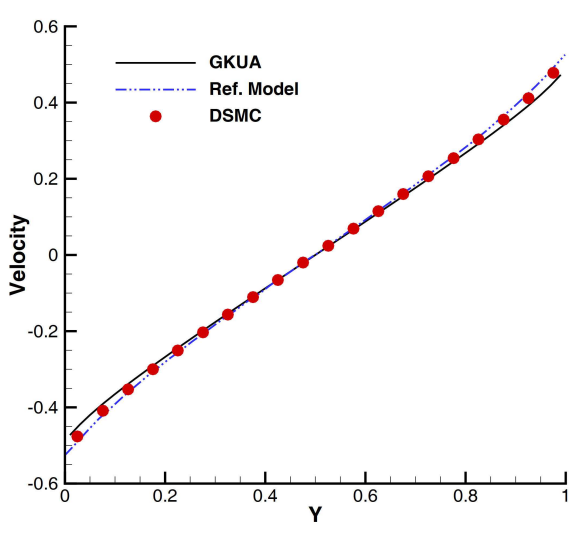

(a) Tangential velocity

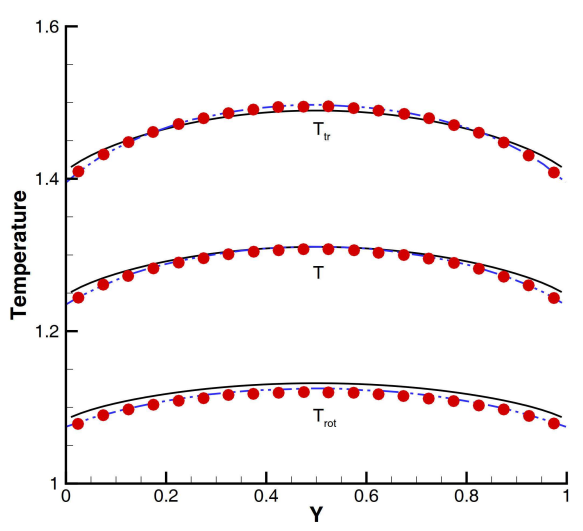

(b) Temperature

Figure 13: Comparisons between the GKUA (lines), model in Ref. [12] (double dot dash lines) and DSMC simulations (circles) for planar Couette flows with methane gases at $K n=1.0$.

the methane gas with the temperature exponent of the viscosity coefficient $\chi=0.84$. Other parameters are set as $\alpha=1, \omega=0.5, \beta=-0.5$, and $\theta$ is determined by Eq. (2.29), where the rotational collision number is set as $Z=2.023$. Good agreement among the results of the present GKUA, the numerical model in Ref. [12] and the DSMC simulations can be seen in Fig. 13.

\subsection{Supersonic flows past a circular cylinder}

In this part, the steady supersonic flow past a circular cylinder at the free-stream Mach number $M a=2.0$ and Knudsen number $K n=0.1$ is simulated by the present algorithm. In order to validate the compatibility and accuracy of the present algorithm for this simulation, the present GKUA results are compared with the DSMC results. All DSMC simulation results are obtained by the DS2V software [50]. Here, due to symmetry, only half plane on the cylinder is considered and symmetry boundary conditions are employed in the simulations.

In this case, the cylinder radius has a value of $0.01 \mathrm{~m}$, the far-field boundary is set $0.1 \mathrm{~m}$, and the computational domain is meshed with $61 \times 61$ quadrilateral cells, as shown in Fig. 14, coupling with the types of boundaries. The simulated gas is chosen as the nitrogen $N_{2}$, where the molecular reference diameter is $\bar{d}=4.17 \times 10^{-10} \mathrm{~m}$ and the temperature exponent of the viscosity coefficient is $\chi=0.74$. The free-stream has a velocity $U_{\infty}=673.7394 \mathrm{~m} / \mathrm{s}$ with temperature $T_{\infty}=273 \mathrm{~K}$ and molecule number density $n_{\infty}=$ $1.2944 \times 10^{21} / \mathrm{m}^{3}$. The cylinder has a cold surface with a constant temperature $T_{w}=273 \mathrm{~K}$ with diffusive reflection boundary condition. The rotational collision number $Z=5$ in this case. Other parameters are set as $\alpha=1, \omega=0.5, \operatorname{Pr}=0.72, \beta$ and $\theta$ is determined by Eq. (2.29). The modified Gauss-Hermite quadrature rule with $24 \times 24$ discrete velocity 


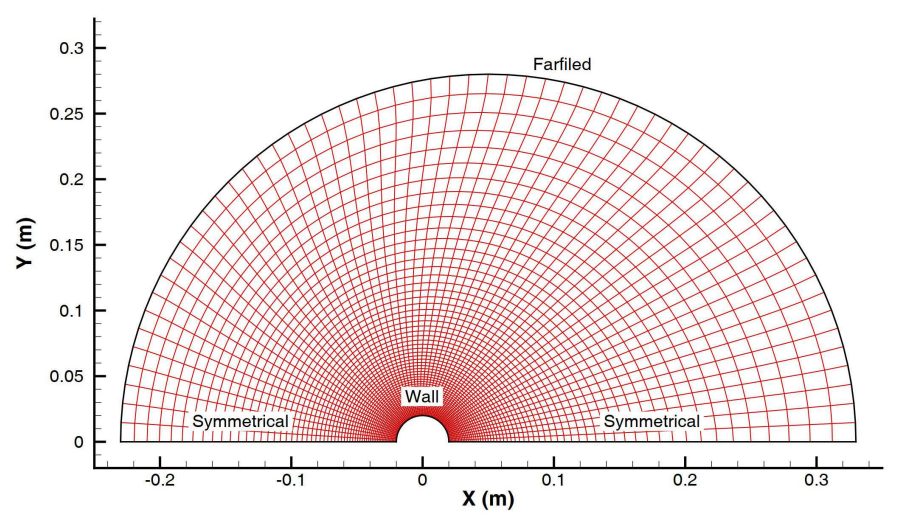

Figure 14: Computational grid system of the supersonic flows past a circular cylinder at $K n=0.1$.

ordinate points is applied for the simulations to evaluate the macroscopic flow moments over the velocity space. The simulation is performed until the residual error of the density is less than $10^{-4}$ or the final time $t=10^{5}$. The LU-SGS implicit scheme is applied in this simulation. The time step is determined by Eq. (3.15), and the CFL number is set as 0.5 initially, and equably increased to 50 .

Fig. 15 presents the flow variables along central symmetric line in front of the stagnation point at $K n=0.1$. It can be found that the GKUA results match well with DSMC solutions in all macroscopic flow variable distributions, which demonstrates the feasibility of the present algorithm for the diatomic gas flow simulations. Besides, the program operates about 3.0 hours, and the required RAM of the distribution functions is $47.461 \mathrm{Mb}$ with double precision.

In addition, the contour lines of the dimensional macro-variables for the monotonic (Ar) and diatomic $\left(N_{2}\right)$ gases under $K n=5 \times 10^{-4}$, which represents the continuum flows, are compared in Fig. 16. For the simulation of $N_{2}$ gas, it uses nearly 11.5 CPU hours, while 12 hours as the $A r$ gas. The required RAMs of the distribution functions for these two gases are the same as the first case.

It can be observed that the position of the shock wave for the $\mathrm{N}_{2}$ gas flow is closer to the surface than it in the $A r$ gas flow, and the overall temperature of $N_{2}$ is quite lower than the $A r$ case in the region behind the shock wave. These phenomena may be caused by the excitation of the internal energy. For the diatomic gas, we think the rotational degrees of freedom are excited fully, which converts partial translational energy into internal energy. While there only considers the translational energy in the monotonic gas, leads to a higher temperature distribution in the region behind the shock wave than the diatomic gas case. 


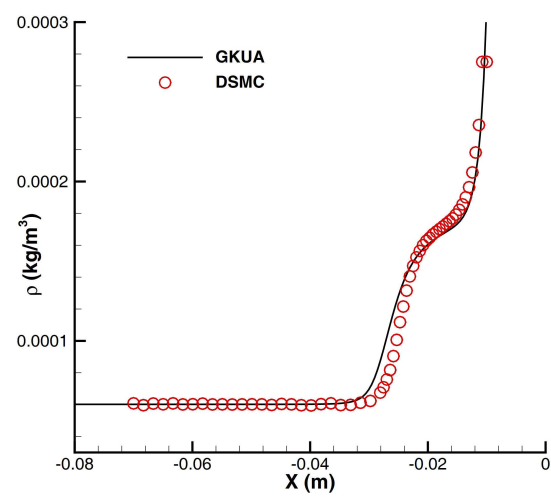

(a) Density

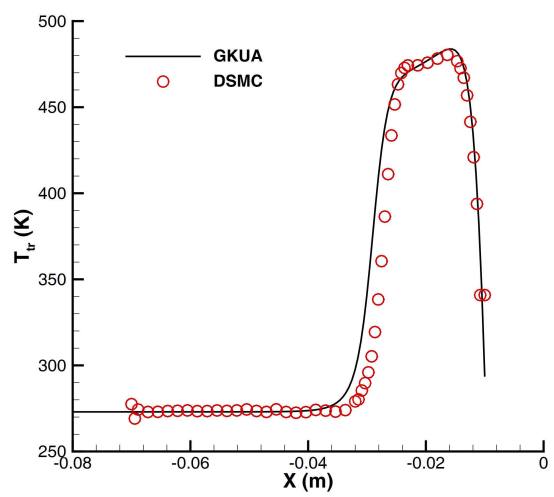

(c) Translational temperature

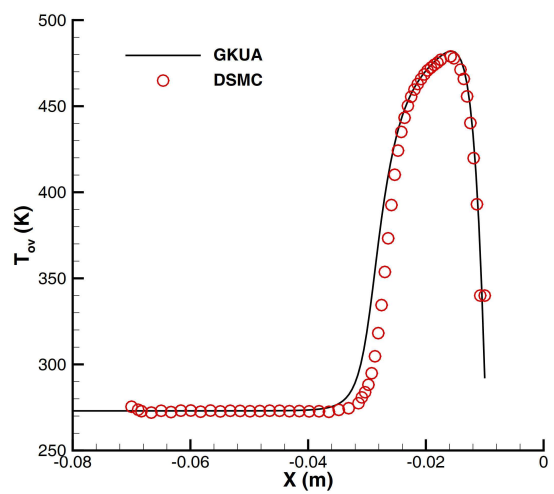

(e) Overall temperature

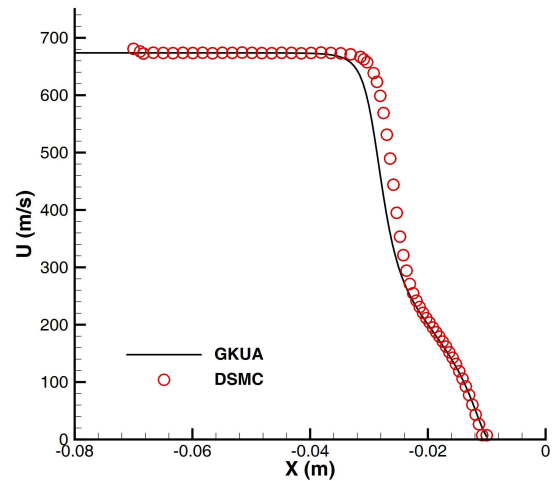

(b) $U$-velocity

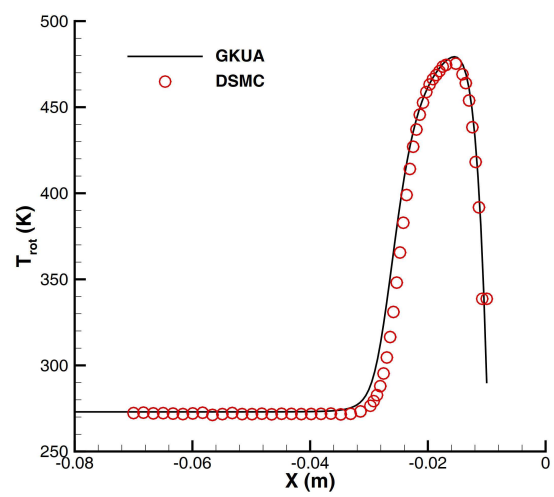

(d) Rotational temperature

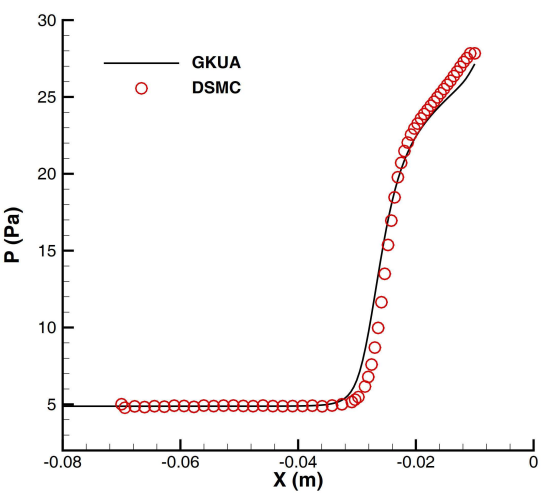

(f) Pressure

Figure 15: Dimensional macro-variable distributions along central symmetric line in front of the stagnation point at $M a=2.0$ and $K n=0.1$ with $N_{2}$ gases obtained by GKUA (line) and DSMC (red circle). 


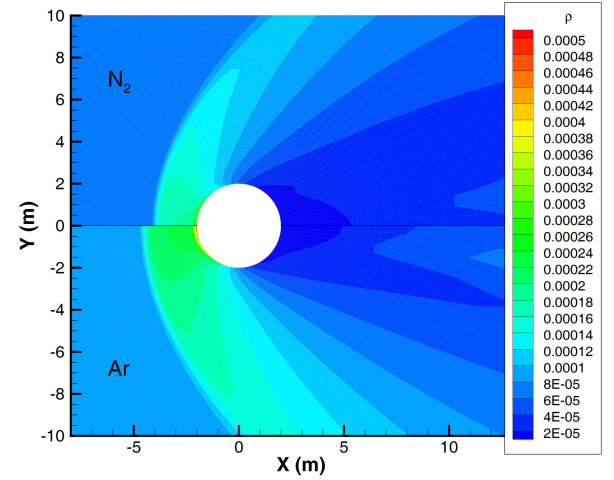

(a) Density

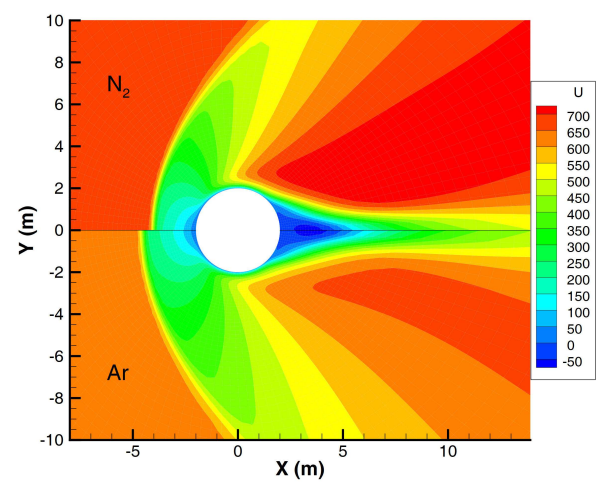

(c) $U$-velocity

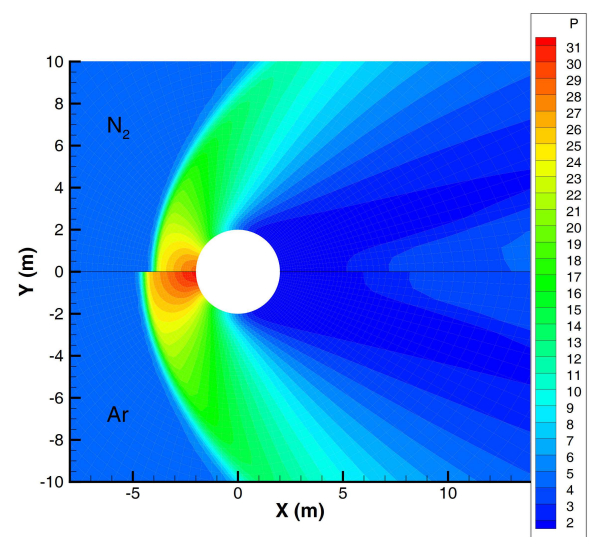

(e) Pressure

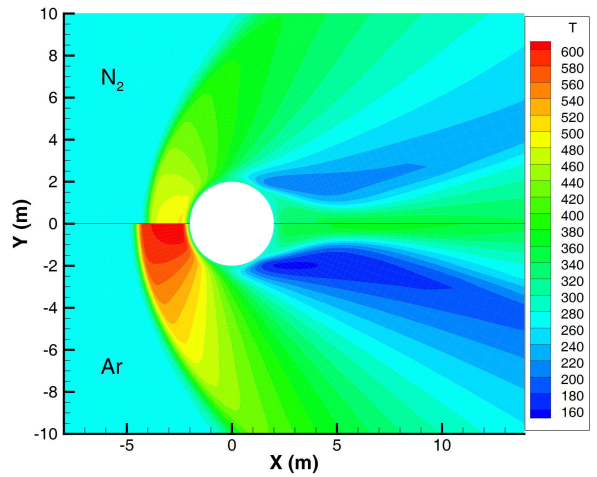

(b) Overall temperature

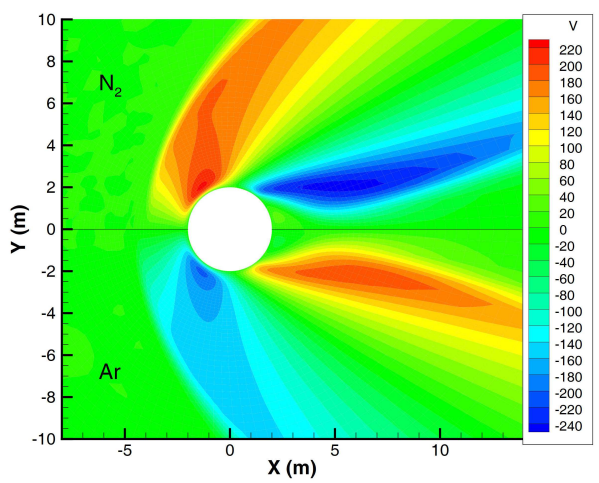

(d) $V$-velocity

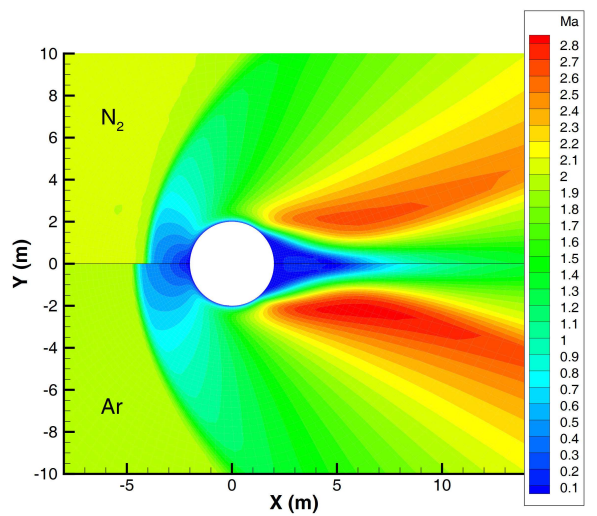

(f) Mach number

Figure 16: Dimensional macro-variable contours for the supersonic flows past a cylinder at $M a=2.0$ and $K n=5 \times 10^{-4}$ for $N_{2}$ (up) and $A r$ (down) gases. 


\subsection{Hypersonic flow around a flat plate placed normally}

Due to the hypersonic velocity, the reentry flight environment of the space vehicles includes shock-shock interactions and shock-boundary interactions that cause high heat transfer and pressure on the body of the spacecraft. Strong thermal non-equilibrium is associated with these flows. It is important to study the physical flow around spacecraft in a hypersonic rarefied environment in order to understand flow phenomenon and to design a real size vehicle.

The hypersonic rarefied gas flow past an spacecraft-like solar panel (strip) is a fundamental problem in reentry aerodynamics. Here, the flow around a reentry flat plate placed normally for the diatomic gas will be simulated by the present gas-kinetic unified algorithm, where highly non-equilibrium between translational and rotational temperatures appears around the plate.

The inflow nitrogen gas has a velocity $U_{\infty}=610.526 \mathrm{~m} / \mathrm{s}$ with the temperature $T_{\infty}=$ $12.81 \mathrm{~K}$, whose Mach number is $M a=8.3666$, correspondingly. The ratio of the wall temperature to the total temperature is set as $T_{w} / T_{o}=2 / 3$, which can obtain that the dimensionless wall temperature $T_{w}=10.0$. The viscosity-temperature index $\chi=0.74$, and other parameters are set as $\alpha=1, \omega=0.5, \beta=-0.5$ and $\theta$ is determined by Eq. (2.29). Due to symmetry, only half plane on the flat plate is considered and symmetry boundary conditions were employed, where the computational domain is meshed with $63 \times 51$ quadrilateral cells, as shown in Fig. 17, coupling with the types of boundaries. The GaussChebyshev integral rule is applied in this simulation, where the domain in velocity space $\left(V_{x}, V_{y}\right) \in[(-15,15) \times(-12,12)]$ is discretized with $70 \times 50$ mesh points.

Fig. 18 presents the distributions of the dimensionless density, translational and rotational temperatures on the deceleration line $y=0$ ahead of the plate for $K n=0.1$, comparing with the results in Ref. [51]. For this simulation, the program operates about 4.0 hours, and the required RAM of the distribution functions is $248.337 \mathrm{Mb}$ with double precision.

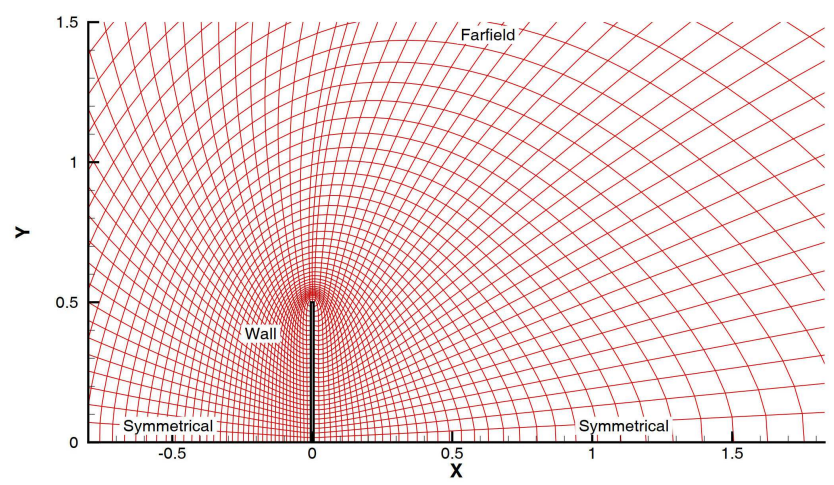

Figure 17: Computational grid system of the hypersonic flow around an infinite flat plate placed normally. 


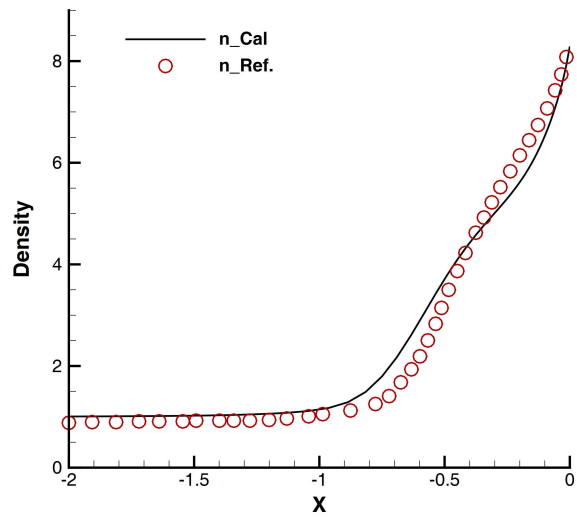

(a) Density

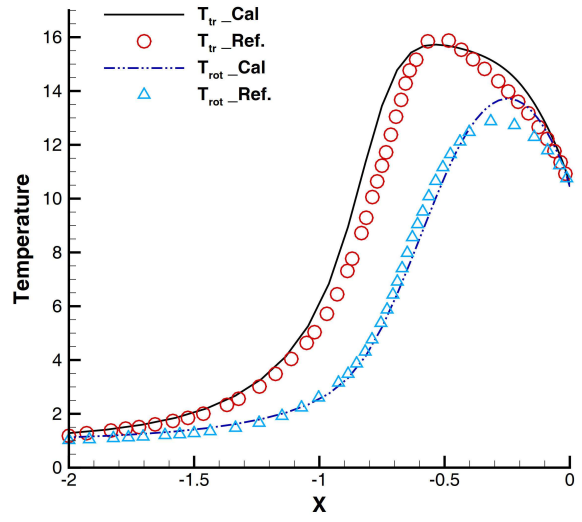

(b) Translational and rotational temperatures

Figure 18: Distributions on the deceleration line $y=0$ ahead of the plate for $K n=0.1$.

It can be observed that the present GKUA results match well with the results in the reference. Besides, we can find that there exist one detached shock wave, or called the strong disturbance, located in the front of the vertical plate along the deceleration line, which causes the high temperature region. Comparing the distributions of the translational and rotational temperatures, it can be found that the maximum value of the dimensionless translational temperature is $T_{t r}=15.719$ approximately, while it is $T_{r o t}=13.708$ in the rotational temperature profile, showing the serious non-equilibrium flow effects presented in the front of the vertical plate.

Furthermore, in Fig. 19, the contour lines from the present GKUA for density, overall temperature, translational temperature, and rotational temperature, Mach number for $K n=0.1$ are plotted together, as well as the streamlines in the adjacent region of the plate. Fig. 20 presents the density, overall temperature, translational temperature, and rotational temperature, Mach number contours for $K n=0.01$, as well as the streamlines. Comparing Fig. 19 with Fig. 20, it can be found that the detached shock wave in the flow field of $K n=0.01$ is more identifiable than that of $K n=0.1$, which indicates that the flow is transforming from the rarefied non-equilibrium flow of $K n=0.1$ with strong disturbance shock layer shown in Fig. 19 into the near-continuum flow of $K n=0.01$ with clear detached shock wave and leeward wake separation zone shown in Fig. 20. For this simulation, it uses nearly 5.5 CPU hours, and the required RAM of the distribution functions is the same as the first case.

From the distributions of the translational and rotational temperature at $K n=0.01$ in near-continuum flow regime, we can find that these two contour lines of temperature are almost the same, indicating that the flow field tends to the equilibrium state of the gas molecular velocity distribution function. However, for the rarefied flow, i.e., $K n=$ 0.1 , it can be observed that the translational and rotational temperature contours exist 


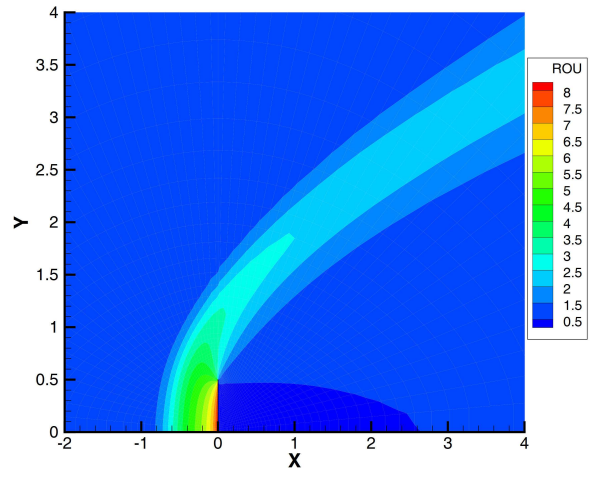

(a) Density

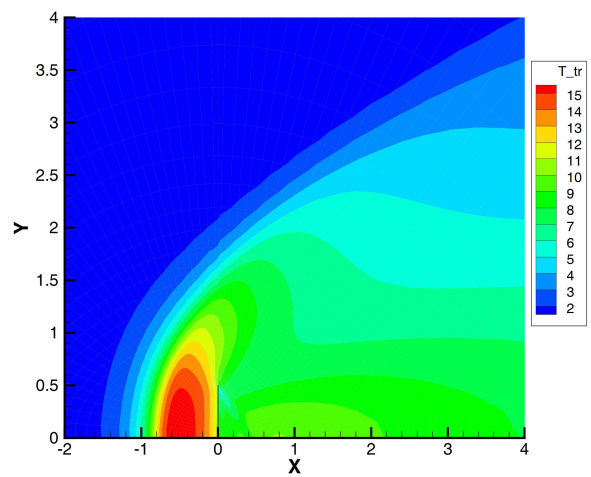

(c) Translational temperature

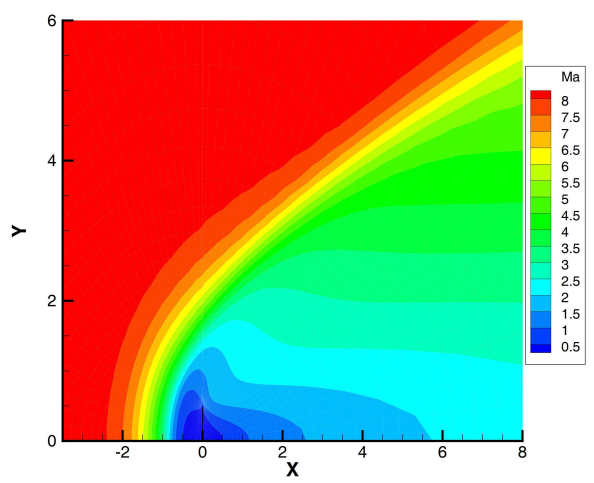

(e) Mach number

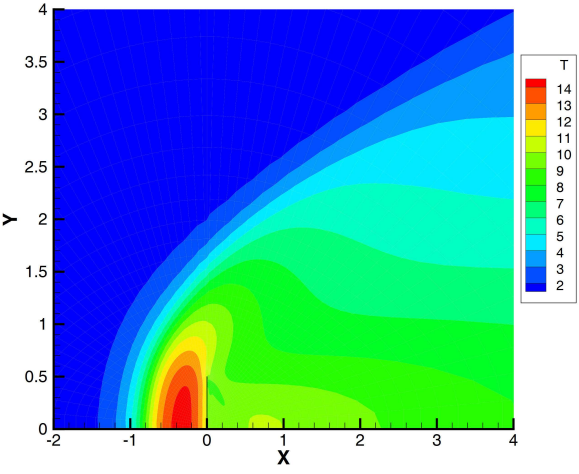

(b) Overall temperature

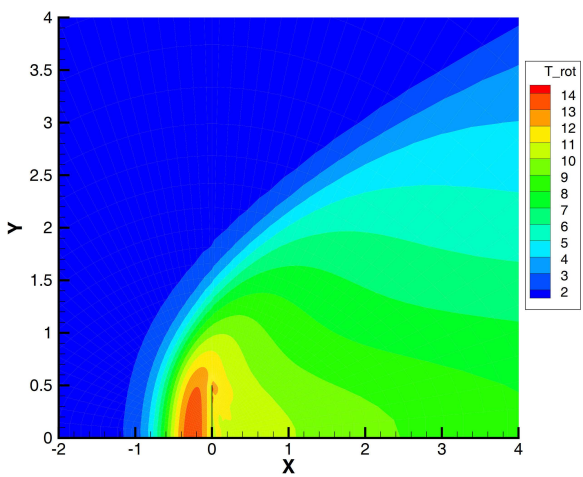

(d) Rotational temperature

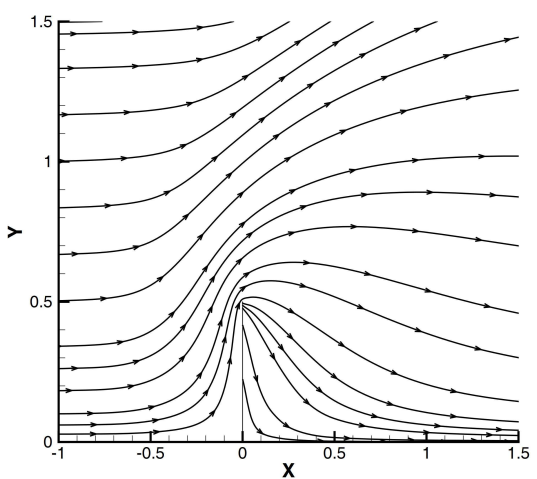

(f) Stream lines

Figure 19: Macro-variable contours or stream lines around the plate for $K n=0.1$. 


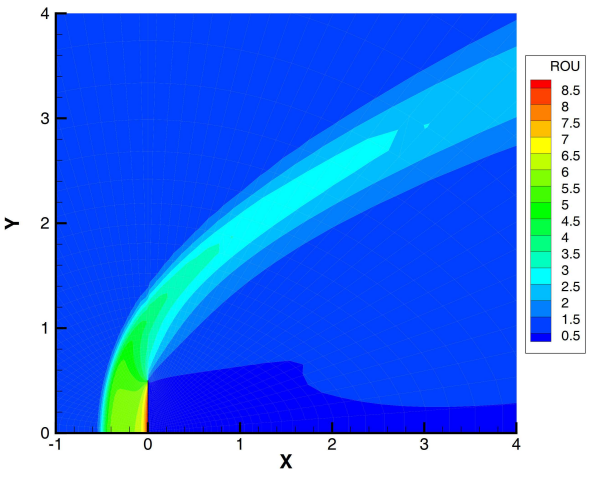

(a) Density

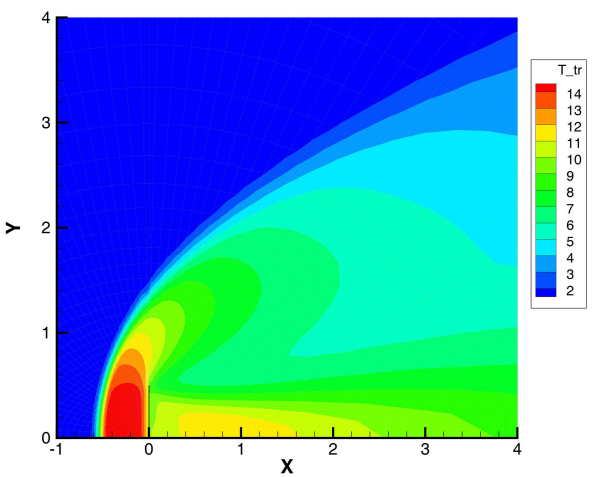

(c) Translational temperature

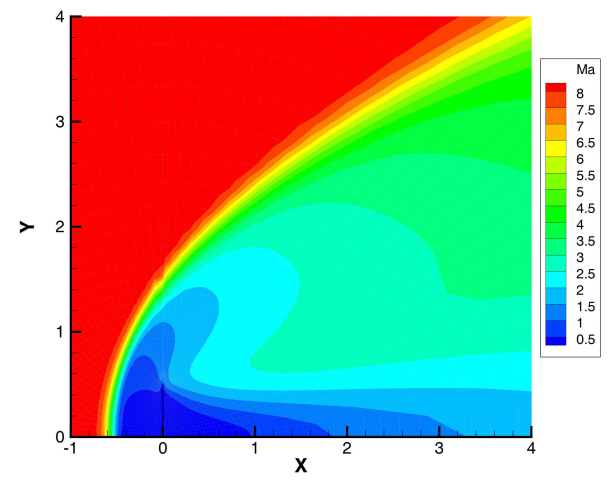

(e) Mach number

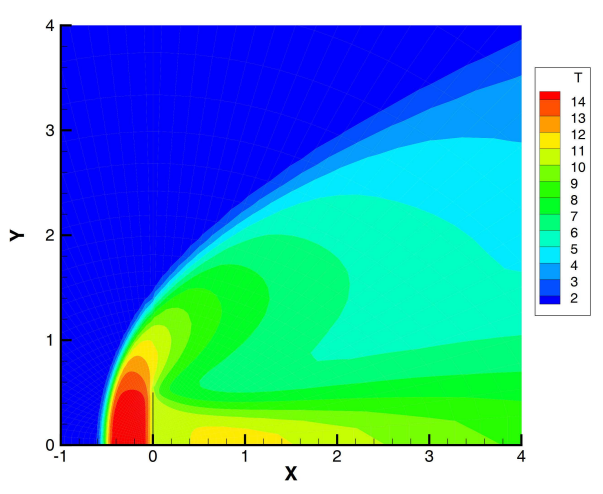

(b) Overall temperature

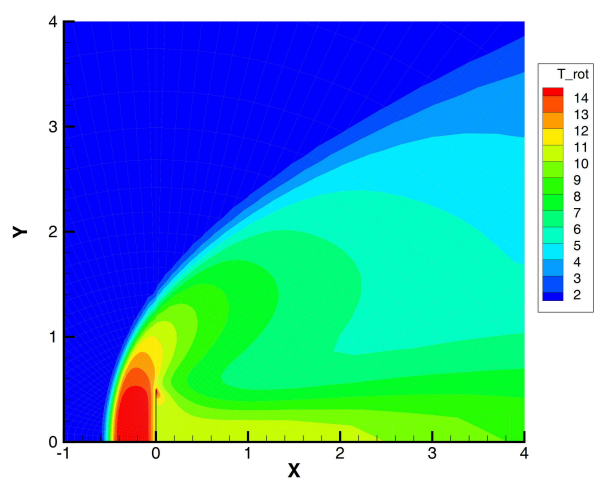

(d) Rotational temperature

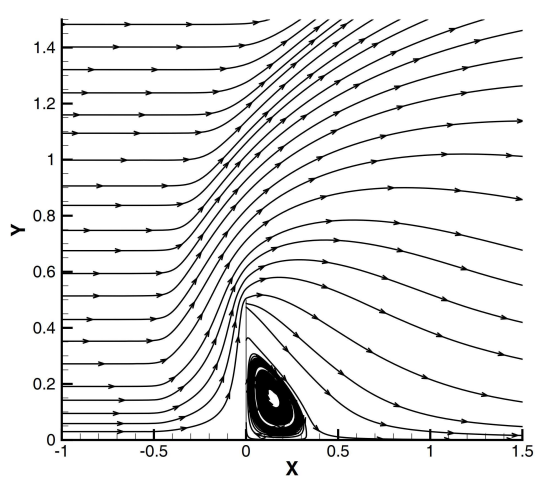

(f) Stream lines

Figure 20: Macro-variable contours or stream lines around the plate for $K n=0.01$. 
significant difference, the gas flow attaches to the surface of the plate accompanied by severe velocity slip and temperature jump, and transits to the downstream flow state smoothly, which do not develop the recirculation region. While for the flow at $K n=$ 0.01 , the recirculation region arises in the backward plate visibly, which can be shown intuitively in Fig. 20(f).

\section{Concluding remarks}

In this paper, based on the ellipsoidal statistical model with rotational energy excitation, the gas-kinetic unified algorithm covering various flow regimes has been developed for the polyatomic gas flows in the rotational non-equilibrium state. Unifying expressions on the gas molecular collision relaxing parameter and the local equilibrium distribution function have been constructed, which are mainly related with the macroscopic flow variables, the flow state controlling parameter $K n$, and the molecular models. The relaxation parameters $\beta$ and $\theta$ have been introduced to define the relaxation temperature and the corrected tensor, and determine the specific expressions of the collision relaxation parameters and the local equilibrium VDFs. Determinations of these two parameters have been given in detail. The corresponding CDVOM has been constructed, which preserves the conservative properties on the mass, momentum and energy during the collision procedure at the discrete level by the numerical method. Utilizing the CDVOM, the unified kinetic modeling equation has been transformed into hyperbolic conservation forms at each of the discrete velocity ordinate points. The explicit and implicit schemes have been developed to solve the discrete equations directly. Besides, according to the conservation of the mass flow flux and energy balance on the surface, the mathematical model and the numerical procedures for the gas-kinetic wall boundary condition in the rotational non-equilibrium state have been developed and presented in detail.

Applying the new version of the GKUA, some selected numerical examples have been investigated to test the present algorithm, including the Sod Riemann problem, homogeneous flow rotational relaxation, normal shock structure calculations, planar Fourier and Couette flows, supersonic flows past a circular cylinder, and hypersonic flow passing a reentry flat plate placed normally. Comparing with the analytic, experimental, DSMC, and some other models or measurements in the references, it has been found that the numerical simulation results obtained by the present algorithm match well in agreement, which demonstrates the validity and accuracy of the present algorithm. Especially, some polyatomic gas non-equilibrium phenomena have been observed and analysed by solving the Boltzmann-type velocity distribution function equation covering various flow regimes.

This work only extend the GKUA as a new version for the polyatomic flows at translational-rotational non-equilibrium state in the near-earth space flight environment, which are preliminary application and computational test for the hypersonic reentry flow covering various flow regimes. Investigations on complex three-dimensional hypersonic 
flows of the vibrational-chemical excited non-equilibrium are needed to get further developments, which may be presented in the future works.

\section{Acknowledgments}

This work is supported by the Project of manned space engineering technology(201814) "Large-scale parallel computation of aerodynamic problems of irregular spacecraft reentry covering various flow regimes", and the National Natural Science Foundation of China (91530319). The authors are thankful to the editors and reviewers for their valuable comments and suggestions, which greatly improved the quality of the manuscript.

\section{References}

[1] H. S. Tsien, Superaerodynamics, mechanics of rarefied gases, Journal of Aeronautics Science 13 (1946) 653-664.

[2] A. P. Peng, Z. H. Li, J. L. Wu and X. Y. Jiang, Validation and analysis of gas-kinetic unified algorithm for solving Boltzmann model equation with vibrational energy excitation, Acta Phys. Sin. 66 (2017) 204703-17.

[3] S. Yoon, P. Gnoffo, J. White and J. Thomas, Computational challenges in hypersonic flow simulations, 39th AIAA Thermophysics Conference (2007) 4265.

[4] Z. H. Li, A. P. Peng, H. X. Zhang and J. Y. Yang, Rarefied gas flow simulations using highorder gas-kinetic unified algorithms for Boltzmann model equations, Prog. Aerosp. Sci. $\mathbf{7 4}$ (2015) 81-113.

[5] Z. H. Li, H. X. Zhang, Study on Gas Kinetic Algorithm for Flows from Rarefied Transition to Continuum, Proc. of 22nd International Symposium on Rarefied Gas Dynamics, ed. by Bartel T.J. and Gallis M.A., pp. 628-636, Sydney, Australia, 2000.

[6] J. Liang, Z. H. Li, X. G. Li and W. B. Shi, Monte Carlo simulation of spacecraft reentry aerothermodynamics and analysis for ablating disintegration, Commun. Comput. Phys. 23 (2018) 1037-1051.

[7] R. Votta, A. Schettino and A. Bonfiglioli, Hypersonic high altitude aerothermodynamics of a space re-entry vehicle, Aerosp. Sci. Technol. 25 (2013) 253-265.

[8] G. Bird, The Q-K model for gas-phase chemical reaction rates, Phys. Fluids 23 (2011) 10610113.

[9] A. A. Shevyrin, P. V. Vashchenkov, Y. A. Bondar and M. S. Ivanov, Validation of DSMC Results for Chemically Nonequilibrium Air Flows Against Measurements of the Electron Number Density in RAM-C II Flight Experiment, Proceedings of the 29th International Symposium on Rarefied Gas Dynamics (2014) 155-161.

[10] S. Chapman and T. G. Cowling, The Mathematical Theory of Non-Uniform Gases, $3^{\text {rd }}$ Ed., Cambridge University Press (1970).

[11] C. Cercignani, Mathematical Methods in Kinetic Theory, Revised Edition, Plenum Press, New York (1990).

[12] L. Wu, C. White, T. J. Scanlon, J. M. Reese and Y. H. Zhang, A kinetic model of the Boltzmann equation for non-vibrating polyatomic gases, J. Fluid Mech. 763 (2015) 24-50.

[13] B. Rahimi and H. Struchtrup, Macroscopic and kinetic modelling of rarefied polyatomic gases, J. Fluid Mech. 806 (2016) 437-505. 
[14] C. S. Wang-Chang and G. E. Uhlenbeck, Transport phenomena in polyatomic gases, Research Rep. CM-681, University of Michigan Engineering (1951).

[15] P. L. Bhatnagar, E. P. Gross and M. Krook, A model for collision processes in gases. I. Small amplitude processes in charged and neutral one-component systems, Phys. Rev. 94 (1954) 511.

[16] E. M. Shakhov, Generalization of the Krook kinetic relaxation equation, Fluid Dynam. 3 (1968) 95-96.

[17] L. H. Holway, New statistical models for kinetic theory: Methods of construction, Phys. Fluids 9 (1966) 1658-1673.

[18] V. A. Rykov, Model Kinetic equation of a gas with rotational degrees of freedom, Fluids Dyn. 10 (1975) 959-966.

[19] P. Andries, P. L. Tallec, J. P. Perlat and B. Perthame, The Gaussian-BGK model of Boltzmann equation with small Prandtl number, Eur. J. Mech. B - Fluids 19 (2000) 813-830.

[20] S. Brull and J. Schneider, On the ellipsoidal statistical model for polyatomic gases, Continuum Mech. Thermodyn. 20 (2009) 489-508.

[21] F. Bernard, A. Iollo and G. Puppo, BGK polyatomic model for rarefied flows, J. Sci. Comput. 78 (2019) 1893-1916.

[22] Y. Dauvois, J. Mathiaud and L. Mieussens, An ES-BGK model for vibrational polyatomic gases, arXiv preprint (2020) arXiv:2007.02727.

[23] C. Klingenberg, M. Pirner and G. Puppo, A consistent kinetic model for a two-component mixture of polyatomic molecules, arXiv preprint (2018) arXiv:1806.11486.

[24] J. T. Huang, W. A. Yong, Boundary conditions of the lattice Boltzmann method for convection-diffusion equations, J. Comput. Phys. 300 (2015) 70-91.

[25] Z. H. Chai, B. C. Shi and Z. L. Guo, A multiple-relaxation-time lattice Boltzmann model for general nonlinear anisotropic convection-diffusion equations, J. Sci. Comput. 69 (2015) 355-390.

[26] L. Wang, Z. H. Chai and B. C. Shi, Regularized lattice Boltzmann simulation of doublediffusive convection of power-law nanofluids in rectangular enclosures, Int. J. Heat Mass Transf. 102 (2016) 381-395.

[27] J. C. Mandal and S. M. Deshpande, Kinetic flux vector splitting for euler equations, Comput. Fluids 23 (1994) 447-478.

[28] K. Xu and Z. H. Li, Microchannel flow in the slip regime: gas-kinetic BGK-Burnett solutions, J. Fluid Mech. 513 (2004) 87-110.

[29] X. Ji and K. Xu, Performance enhancement for high-order gas-kinetic scheme based on WENO-adaptive-order reconstruction, Commun. Comput. Phys. 27 (2020) 145-166.

[30] Q. B. Li, An improved gas-kinetic scheme for multimaterial flows, Commun. Comput. Phys. 28 (2020) 539-590.

[31] W. Q. Hu and Z. H. Li, Investigation on different discrete velocity quadrature rules in gaskinetic unified algorithm solving Boltzmann model equation, Comput. Math. Appl. 75 (2018) 4179-4200.

[32] Z. H. Li, W. Q. Hu, A. P. Peng, J. J. Wu and C. H. Lee, Gas-kinetic unified algorithm for plane external force-driven flows covering all flow regimes by modeling of Boltzmann equation, Int. J. Numer. Meth. Fluids 92 (2020) 922-949.

[33] X. J. Gu and D. R. Emerson, A high-order moment approach for capturing non-equilibrium phenomena in the transition regime, J. Fluid Mech. 636 (2009) 177-216.

[34] S. Liu, P. B. Yu, K. Xu and C. W. Zhong, Unified gas-kinetic scheme for diatomic molecular simulations in all flow regimes, J. Comput. Phys. 259 (2014) 96-113. 
[35] D. X. Pan, C. W. Zhong and C. S. Zhuo, An implicit discrete unified gas-kinetic scheme for simulations of steady flow in all flow regimes, Commun. Comput. Phys. 25 (2019) 1469-1495.

[36] A. P. Peng, Z. H. Li, J. L. Wu, and X. Y. Jiang, Implicit gas-kinetic unified algorithm based on multi-block docking grid for multi-body reentry flows covering all flow regimes, J. Comput. Phys., 327 (2016) 919-942.

[37] S.-M. Hou, Z.-H. Li, X.-Y. Jiang and S. Zeng, Numerical study on two-dimensional microchannel flows using the gas-kinetic unified algorithm, Commun. Comput. Phys. 23 (2018) 1393-1414.

[38] A. B. Huang and D. P. Giddens, The Discrete Ordinate Method for the Linearized Boundary Value Problems in Kinetic Theory of Gases. In: Proceedings of 5th International Symposium on Rarefied Gas Dynamics, edited by C. L. Brundin, New York, Vol. 1, pp. 481-486, 1967.

[39] B. Shizgal, A Gaussian quadrature procedure for use in the solution of the Boltzmann equation and related problems, J. Comput. Phys. 41 (1981) 309-328.

[40] D. Goldstein, B. Sturtevant and J. E. Broadwell, Investigations of the motion of discrete velocity gases, Progress in Astronautics and Aeronautics 117 (1989) 100-117.

[41] W. Q. Hu, Y. T. Gao, Z. Z. Lan, C. Q. Su and Y. J. Feng, Lattice Boltzmann model for a generalized Gardner equation with time-dependent variable coefficients, Appl. Math. Model. 46 (2017) 126-140.

[42] K. Koura and H. Matsumotoa, Variable soft sphere molecular model for air species, Phys. Fluids A: Fluid Dynam. 4 (1992) 1083-1085.

[43] G. A. Bird, Monte-Carlo simulation in an engineering context, Prog. Astronaut. Aeronaut. 74 (1981) 239-255.

[44] V. A. Titarev, Conservative numerical methods for model kinetic equations, Comput. Fluids, 36 (2007) 1446-1459.

[45] B. V. Leer, Towards the ultimate conservative difference scheme. II. Monotonicity and conservation combined in a second-order scheme, J. Comput. Phys. 14 (1974) 361-370.

[46] J. Blazek, Computational Fluid Dynamics: Principles and Applications, ButterworthHeinemann (2015).

[47] S. M. Cox, and P. C. Matthews, Exponential time differencing for stiff systems, J. Comput. Phys. 176 (2002) 430-455.

[48] G. S. Jiang and C. W. Shu, Efficient Implementation of Weighted ENO Schemes, J. Comput. Phys. 126 (1996) 201-228.

[49] H. Alsmeyer, Density profiles in argon and nitrogen shock waves measured by the absorption of an electron beam, J. Fluid. Mech. 74 (1976) 497-513.

[50] G. A. Bird, The DS2V /3V program suite for DSMC caculations, Proceedings of the 24th International Symposium on Rarefied Gas Dynamics (2005) 541-546.

[51] V. A. Rykov, V. A. Titarev and E. M. Shakhov, Numerical study of the transverse supersonic flow of a diatomic rarefied gas past a plate, Comput. Math. Math. Phys. 47 (2007) 136-150. 